\title{
WR bubbles and He II emission ${ }^{\star}$
}

\author{
Y. Nazé ${ }^{1, \star \star}$, G. Rauw ${ }^{1, \star \star \star}$, J. Manfroid ${ }^{1, \dagger}$, Y.-H. Chu ${ }^{2}$, and J.-M. Vreux ${ }^{1}$ \\ ${ }^{1}$ Institut d'Astrophysique et de Géophysique, Université de Liège, Allée du 6 Août 17, Bât. B5c, 4000 Liège, Belgium \\ 2 Astronomy Department, University of Illinois at Urbana-Champaign, 1002 West Green Street, Urbana, IL 61801, USA
}

Received 25 March 2003 / Accepted 20 May 2003

\begin{abstract}
We present the very first high quality images of the He II $\lambda 4686$ emission in three high excitation nebulae of the Magellanic Clouds. A fourth high excitation nebula, situated around the WR star BAT99-2, was analysed in a previous letter. Using VLT FORS data, we investigate the morphology of the ring nebulae around the early-type WN stars BAT99-49 \& AB7. We derive the total He II fluxes for each object and compare them with the most recent theoretical WR models. Whilst the ionization of the nebula around BAT99-49 can be explained by a WN star of temperature 90-100 kK, we find that the $\mathrm{He}$ II emission measure of the nebula associated with $\mathrm{AB} 7$ requires an $\mathrm{He}^{+}$ionizing flux larger than predicted for the hottest WN model available. Using $\mathrm{H} \alpha$, [O III] and $\mathrm{He}$ I $\lambda 5876$ images along with long-slit spectroscopy, we investigate the physical properties of these ring nebulae and find only moderate chemical enrichment.

We also surveyed seven other LMC WR stars but we failed to detect any He II emission. This holds also true for BAT99-9 which had been proposed to excite an He II nebula. Four of these surveyed stars are surrounded by a ring nebula, and we use the FORS data to derive their chemical composition: the nebula around BAT99-11 shows a N/O ratio and an oxygen abundance slightly lower than the LMC values, while the nebula around BAT99-134 presents moderate chemical enrichment similar to the one seen near BAT99-2, 49 and AB7. Comparing the WR stars of the LMC, BAT99-2 and 49 appear unique since similar stars do not reveal high excitation features.

The third high excitation nebula presented in this paper, $\mathrm{N} 44 \mathrm{C}$, does not harbor stars hotter than mid-O main sequence stars. It was suggested to be a fossil X-ray nebula ionized by the transient LMC X-5. Our observations of N44C reveal no substantial changes in the excitation compared to previous results reported in the literature. Therefore, we conclude that either the recombination timescale of the X-ray nebula has been underestimated or that the excitation of the nebula is produced by another, yet unknown, mechanism.
\end{abstract}

Key words. Wolf-Rayet - ISM: individual object: LMC N44C - HII regions - ISM: bubbles - ISM: abundances - Magellanic Clouds

\section{Introduction}

Massive stars are known to possess strong stellar winds. Throughout their lives, these winds are able to shape their environment. Winds of $\mathrm{O}$ stars can sweep up the Interstellar Medium (ISM) and create interstellar bubbles, a few examples of which have been given in Nazé et al. (2001, 2002). While evolving into a Luminous Blue Variable or a Red Supergiant phase, the star blows a very dense and slow wind that can be swept up by the subsequent faster Wolf-Rayet (WR) wind. This second wind-blown bubble will ultimately collide with the first one. Numerical simulations of such processes have been performed by e.g. García-Segura et al. (1996, and references therein).

Send offprint requests to: Y. Nazé, e-mail: naze@astro.ulg.ac.be

* Based on observations collected at the European Southern Observatory, Cerro Paranal, Chile (ESO No. 68.C-0238(A,B)).

$\star \star$ Research Fellow FNRS (Belgium).

$\star \star \star$ Research Associate FNRS (Belgium).

$\dagger$ Research Director FNRS (Belgium).
Many examples of ring nebulae around WR stars are known, both in the Galaxy and in the Magellanic Clouds (MCs). However, the exact nature and evolutionary status of these candidate bubbles are still unknown in most cases, but could be investigated by means of kinematic studies (e.g. Chu et al. 1999) and/or abundance analyses.

A few WR ring nebulae present an additional, peculiar feature: nebular He II $\lambda 4686$ emission. Such a high excitation feature is common amongst Planetary Nebulae, but $\mathrm{O}$ and WR stars were often thought unable to ionize $\mathrm{He}^{+}$in a detectable manner in their surroundings. Only $7 \mathrm{He}$ II nebulae are known in the Local Group (Garnett et al. 1991a), and five of them are around WR stars. Nebular He II features have also been found farther away, in starburst galaxies, and were again attributed to WR stars (Schaerer 1996), though this interpretation is now questioned by Smith et al. (2002). Observations of the He II emission can provide an accurate constraint on the otherwise unobservable far UV fluxes of WR stars, but for the moment, this can only be done with precision for the Local Group objects. 
Table 1. Total exposure times (in s) for each object, in each filter and for each grism. Spectral types are taken from Foellmi et al. (2003a and 2003b), Bartzakos et al. (2001) and Niemela et al. (2002).

\begin{tabular}{|c|c|c|c|c|c|c|c|c|c|}
\hline \multirow[t]{2}{*}{ Object } & \multirow[t]{2}{*}{ Sp. Type } & \multicolumn{6}{|c|}{ Imaging Filters } & \multicolumn{2}{|c|}{ Grisms } \\
\hline & & $\mathrm{H} \alpha$ & [O III] & He I $\lambda 5876$ & He II $\lambda 4686$ & cont. 5300 & cont. 6665 & $600 \mathrm{~B}$ & $600 \mathrm{~V}$ \\
\hline LMC BAT99-2 & WN2b(h) & 300 & 300 & 3600 & 3600 & 540 & 300 & 900 & 900 \\
\hline LMC BAT99-49 & $\mathrm{WN} 4: \mathrm{b}+\mathrm{O} 8 \mathrm{~V}$ & 300 & 300 & 2700 & 2700 & 405 & 300 & 900 & 900 \\
\hline SMC AB7 & WN2-4+O6If & 300 & 300 & 2400 & 2800 & 240 & 300 & 900 & 900 \\
\hline LMC N44C & & 300 & 300 & 1350 & 1400 & 198 & 300 & 500 & 600 \\
\hline LMC BAT99-8 & WC4 & & & & & & & 300 & 100 \\
\hline LMC BAT99-9 & WC4 & & & & & & & 300 & \\
\hline LMC BAT99-11 & WC4 & & & & & & & 300 & 300 \\
\hline LMC BAT99-52 & WC4 & & & & & & & 300 & \\
\hline LMC BAT99-63 & WN4ha: & & & & & & & 300 & 200 \\
\hline LMC BAT99-84 & WC4 & & & & & & & 300 & \\
\hline LMC BAT99-134 & WN4b & & & & & & & 300 & 300 \\
\hline
\end{tabular}

${ }^{a}$ See Paper I.

We thus decided to investigate the case of the three high excitation nebulae around WR stars in the MCs. The nebula around BAT99-2 was already analysed in Nazé et al. (2003, hereafter Paper I) and we discuss here the two remaining cases. To date, only low resolution, low signal/noise He II images of the nebulae surrounding BAT99-2 and AB7 exist (Melnick \& Heydari-Malayeri 1991) and we present here the very first high quality VLT images of the high excitation nebulae associated with BAT99-49 and AB7. In addition, we will also investigate a fourth peculiar He II nebula, N44C, which may be a fossil $\mathrm{X}$-ray nebula. All these objects are situated in the LMC, except for AB7, which is in the SMC. Finally, we also present the results of a small survey of LMC WR stars to search for the possible existence of nebular He II emission. Since some of these stars have ring nebulae around them, our spectroscopic data also enable us to examine their physical properties. In this paper, we will first describe the observations, then explore the morphology and spectrophotometry of the high excitation nebulae and present our small WR survey. We will finally conclude in Sect. 5.

\section{Observations}

We obtained CCD images of the He II emission nebulae in the MCs with the FORS1 instrument on the $8 \mathrm{~m}$ VLT-UT3 in 2002 January. The images were taken through several filters: $\mathrm{H} \alpha$ $\left(\lambda_{0}=6563 \AA, F W H M=61 \AA\right),\left[\mathrm{O}\right.$ III] $\left(\lambda_{0}=5001 \AA, F W H M=\right.$ $57 \AA)$, He I $\lambda 5876\left(\lambda_{0}=5866 \AA, F W H M=60 \AA\right)$, He II $\lambda 4686$ $\left(\lambda_{0}=4684 \AA, F W H M=66 \AA\right)$, plus continuum filters centered at $5300 \AA(F W H M=250 \AA)$ and $6665 \AA(F W H M=65 \AA)$. To avoid saturation, we split the observations into multiple, short exposures: the total exposure times achieved are presented in Table 1. The pixel size of the detector is $0 .{ }^{\prime} 2$ on the sky and the seeing was $\sim 1^{\prime \prime}$, thus providing the highest resolution images so far of these nebulae in this filter set. The data were reduced with IRAF $^{1}$ using standard methods for overscan and

\footnotetext{
1 IRAF is distributed by the National Optical Astronomy Observatories, which are operated by the Association of Universities for Research in Astronomy, Inc., under cooperative agreement with the National Science Foundation.
}

bias subtraction and flatfielding. The images of each filter were then aligned and combined with IRAF. We constructed starfree images in the $\mathrm{H} \alpha$ and [O III] lines by subtracting the images obtained in the neighbouring continuum filters. However, these continuum filters are slightly polluted by faint nebular lines. While these faint lines constitute only small contamination compared to the bright $\mathrm{H} \alpha$ and [O III] lines, they are more problematic when we are dealing with the images corresponding to the filters centered on the fainter He I and He II lines. To obtain star-free He I and He II images, we first constructed with DAOPHOT a list of stars by searching emission features on the $5300 \AA$ continuum image. We then used this list of stars as input for psf-fitting on the He I and He II images. A subtracted image is then built automatically when the photometry of all sources is known. The few remaining faint stars were either removed individually or not considered for flux determinations. Figures 1, 2 and 6 present complete $\mathrm{H} \alpha$ images of BAT99-49, AB7 and N44C. Figures 3 and 7 further show close-ups on BAT99-49 and N44C in the four nebular filters, while Fig. 4 compares the whole VLT field centered on AB7 in each nebular filter. Three color images of the high excitation nebulae are available on the web at http://www.eso.org/outreach/ press-rel/pr-2003/pr-08-03.html

During the same observing run, we also obtained long-slit spectra of these 4 objects with the same instrument used in spectroscopic mode. We also made a small survey to study the environment of seven other WR stars of the LMC: BAT99-8, $9,11,52,63,84$, and 134. We used the 600B and $600 \mathrm{~V}$ grisms to obtain a blue spectrum covering the range 3700 $5600 \AA(R \sim 800)$ and a red spectrum covering $4500-6850 \AA$ $(R \sim 1000)$, respectively. The $1.3^{\prime \prime} \times 6.8^{\prime}$ slit was tilted, with respect to the $\mathrm{N}-\mathrm{S}$ direction, by $45^{\circ}$ for the observations of BAT99-2, 11 , and 134 ; by $55^{\circ}$ for BAT99-63; and by $90^{\circ}$ for AB7. In the remaining cases, the slit was oriented in the $\mathrm{N}-\mathrm{S}$ direction. The spatial resolution was $0.9^{\prime \prime}-1.4^{\prime \prime}$ and the spectral resolution, as mesured from the FWHM of the calibration lines, $7 \AA$. The spectra were reduced and calibrated in a standard way using IRAF. For flux calibration, we observed several standard stars from Oke (1990) and we used the mean atmospheric extinction coefficients for CTIO reduced 


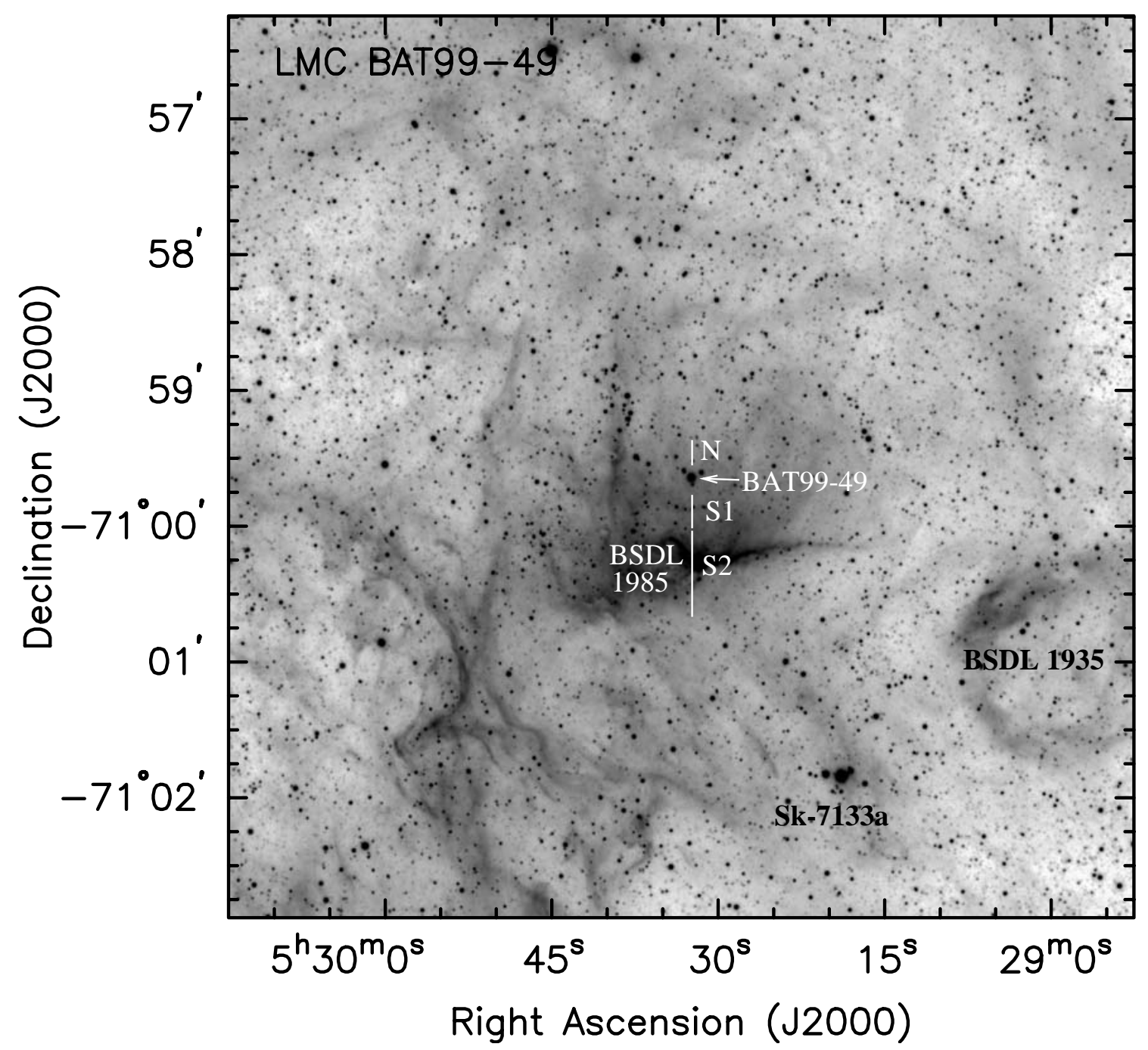

Fig. 1. FORS H $\alpha$ image of LMC BAT99-49. The different regions used for spectral analysis are marked by a solid line, except for the background region, which is just outside the image. Bright stars and features discussed in the text are labelled.

by $15 \%$. We checked that this calibration produces the correct fluxes for the standard stars and for a standard Planetary Nebula (Dopita \& Hua 1997). For the 4 high excitation nebulae, sky subtraction was done using a small region of the spectra where the nebular emission is the lowest. For the WR survey, whenever possible, we used several regions of lowest emission situated on each side of the star and its associated nebula to increase the $\mathrm{S} / \mathrm{N}$. Only a few residuals remained for the brighter sky lines (e.g. [O I] $5577 \AA$ A). The nebular lines were then fitted by Gaussians using SPLOT. The Balmer decrement $\mathrm{H} \alpha / \mathrm{H} \beta$ was used to derive the interstellar extinction, assuming the theoretical case B decrement (Storey \& Hummer 1995) at either $T=10 \mathrm{kK}, 12.5 \mathrm{kK}$ or $15 \mathrm{kK}$, whatever was the closest to the temperature derived using the [O III] lines (see below). Some spectra were contaminated by faint stars, and in such cases, we correct the measured emission line strengths by a $2 \AA$ equivalent width for the Balmer absorptions (McCall et al. 1985) before estimating the reddening. To deredden the line ratios, we used the extinction law from Cardelli et al. (1989) with $R_{\mathrm{V}}=3.1$ for the LMC (Fitzpatrick 1989) and $R_{\mathrm{V}}=2.7$ for the SMC (Bouchet et al. 1985). We used these line ratios in conjunction with the NEBULAR package under IRAF (Shaw \& Dufour 1995) to derive temperatures, densities and abundances, the latter being calculated assuming a constant $n_{\mathrm{e}}=100 \mathrm{~cm}^{-3}$ and $T_{\mathrm{e}}([\mathrm{O} \mathrm{III}])$. If several lines of the same ion exist, the abundance presented in this paper is an average of the abundances derived for each line.

\section{The high excitation nebulae}

\subsection{LMC BAT99-49}

Nebular He II emission was discovered near BAT99-49 (Brey 40a, Sk-7134) by Niemela et al. (1991). This emission apparently covered 70 " on their slit, and corresponds to "a partial ring seen in $\mathrm{H} \alpha$ and [O III]". In a study of kinematics of WR nebulae, Chu et al. (1999) found evidence of interaction between the stellar wind of BAT99-49 and the ambient ISM but did not detect a well-defined expanding shell.

BAT99-49 has been recently re-classified as WN4:b+O8V by Foellmi et al. (2003b). We present in Fig. 1 an $\mathrm{H} \alpha$ image of the whole field, and in Fig. 3 a close-up in the four 


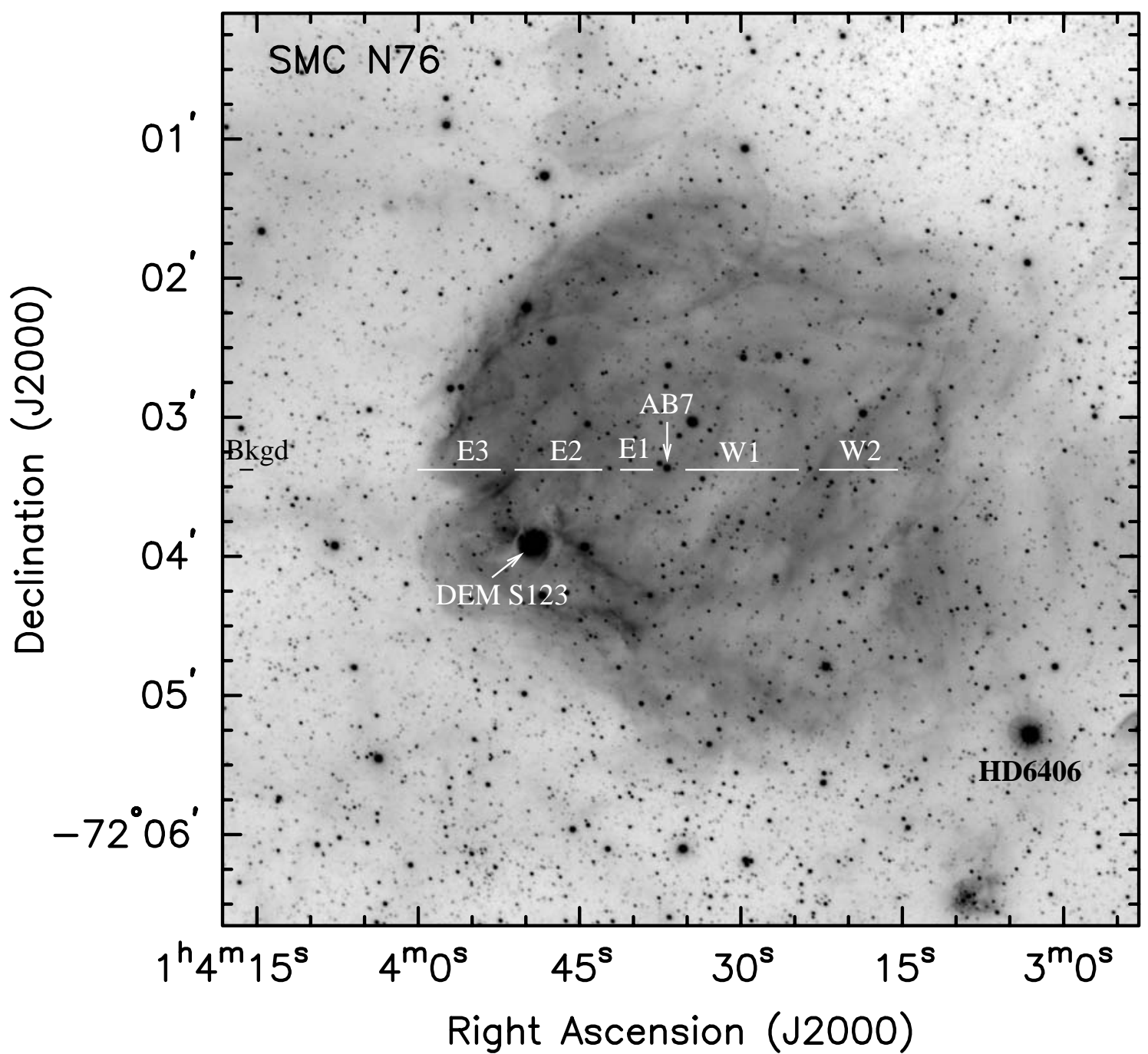

Fig. 2. FORS H $\alpha$ image of SMC N76. The different regions used for spectral analysis are marked by a solid line, and bright stars and features discussed in the text are labelled.

nebular filters. To the south of the star, a small arc of $16^{\prime \prime}$ radius is visible in [O III], and to a lesser extent in $\mathrm{H} \alpha$. A very bright $\mathrm{H} \alpha$ region, BSDL 1985, is also present some $40 "$ south of the star. It was identified as the ring nebula associated with BAT99-49 by Dopita et al. (1994). The southern limit of that region has a lower $[\mathrm{O} \mathrm{III}] / \mathrm{H} \alpha$ ratio than the direct surroundings of BAT99-49. A second $\mathrm{H} \alpha$ region encloses the star to the east, at roughly $36^{\prime \prime}$, but this region completely disappears in [O III]. A beautiful ring nebula, BSDL 1935 (Bica et al. 1999) is present to the southwest of the field and possesses a very low [O III] $/ \mathrm{H} \alpha$ ratio. Generally, the regions to the west of BAT99-49 present a higher $[\mathrm{O}$ III $] / \mathrm{H} \alpha$ ratio than those to the east. As for BAT99-2 (see Paper I), the He I image appears well correlated with $\mathrm{H} \alpha$. On the contrary, the He II image shows completely different features. The brightest He II regions are the small arc 16" south of the star and the bright $\mathrm{H} \alpha$ region $40^{\prime \prime}$ south of the star. A halo surrounds the star and fills the region between the star and the two brighter features. Still fainter emission is also present, but even our $2700 \mathrm{~s}$ exposure just barely detects it. Compared to BAT99-2, the He II emission is much fainter but also more extended.
By comparing the flux in ADU on the images at the position of the slit with our calibrated spectrophotometry convolved with the filter transmission, we were able to calibrate our He II $\lambda 4686$ images. We then measured the He II $\lambda 4686$ flux $^{2}$ in a region $36^{\prime \prime} \times 28^{\prime \prime}$ encompassing the WR star and the brightest He II emission regions. We find a flux of about $1 \times 10^{35} \mathrm{erg} \mathrm{cm}^{-2} \mathrm{~s}^{-1}$ after a reddening correction $A_{\mathrm{V}}$ of 0.4 mag (estimated using the Balmer decrement, see Sect. 2 and Table 2, and assuming a uniform reddening). That corresponds to an ionizing flux of $\sim 1 \times 10^{47}$ photons $\mathrm{s}^{-1}$. At the LMC metallicity $\left(Z=0.2-0.4 Z_{\odot}\right)$, this is what can be expected for a $90-100 \mathrm{kK}$ WN star (Smith et al. 2002). This value may be a lower limit, since we did not take into account all the faintest emission, but its contribution should be rather low. With only $5 \times 10^{36} \mathrm{He}^{+}$ionizing photons s${ }^{-1}$ (Smith et al. 2002), the O8V companion of the WR star has a negligible influence on the He II nebula.

\footnotetext{
${ }^{2}$ Due to errors in the flux calibration and to possible contamination from a poor subtraction of some stars, we estimate that, throughout this paper, the He II fluxes can be considered to have a maximal relative error of $50 \%$.
} 
Table 2. Dereddened line ratios with respect to $\mathrm{H} \beta=100.0$ (see text for details). Uncertainties in the line ratios, estimated from the signal/noise in the lines and the calibration errors, are given in parentheses. The physical properties derived using the line ratios are also indicated below. A ":" denotes an uncertain value, that is not used for abundance calculation when other measurements of the same ion exist.

\begin{tabular}{|c|c|c|c|c|c|c|c|c|}
\hline & \multicolumn{3}{|c|}{ LMC BAT99-49 } & \multicolumn{5}{|c|}{ SMC AB7 } \\
\hline & $\mathrm{N}$ & $\mathrm{S} 1$ & S2 & W1 & W2 & E1 & E2 & E3 \\
\hline [O II] 3727 & $178(19)$ & $244(25)$ & $262(27)$ & $50(5)$ & $121(13)$ & $82(9)$ & $100(11)$ & $251(27)$ \\
\hline H12 & & 9.5: & $5.0(0.5)$ & $6.6(0.7)$ & & $7.7(0.8)$ & $6.5(0.7)$ & $5.4(0.6)$ \\
\hline H11 & & 12: & $6.9(0.7)$ & & & $6.3(0.7)$ & 5. $(0.5)$ & $5.3(0.6)$ \\
\hline H10 & & 10: & $6.9(0.7)$ & $9.4(1)$. & $8.1(0.1)$ & $9.6(1)$. & $7.9(0.8)$ & $8.2(0.9)$ \\
\hline H9 & & 12: & $9.1(0.9)$ & $8.8(0.9)$ & $8.5(0.1)$ & $9.2(1)$. & $8.5(0.9)$ & $7.9(0.8)$ \\
\hline [Ne III] 3868 & $41(4)$ & $44(4)$ & $57(6)$ & $55(6)$ & $55(6)$ & $47(5)$ & $57(6)$ & $59(6)$ \\
\hline $\mathrm{H} 8+\mathrm{He} \mathrm{I}$ & $18(2)$ & $20(2)$ & $20(2)$ & $14(1)$ & $16(2)$ & $17(2)$ & $18(2)$ & $20(2)$ \\
\hline$[\mathrm{Ne} \mathrm{III}]+\mathrm{H} \epsilon$ & $26(2)$ & $27(3)$ & $33(3)$ & $31(3)$ & $29(3)$ & $30(3)$ & $34(3)$ & $35(3)$ \\
\hline $\mathrm{H} \delta$ & $28(2)$ & $29(3)$ & $28(3)$ & $30(3)$ & $29(3)$ & $31(3)$ & $30(3)$ & $30(3)$ \\
\hline $\mathrm{H} \gamma$ & $55(4)$ & $51(4)$ & $50(4)$ & $49(4)$ & $49(4)$ & $50(4)$ & $50(4)$ & $50(4)$ \\
\hline [O III] 4363 & $5.8(0.5)$ & 5. $(0.4)$ & $4.9(0.4)$ & $14(1)$ & $8.9(0.7)$ & $11(1)$ & $13(1)$ & $9.4(0.8)$ \\
\hline He I 4471 & & 2.6: & $4.4(0.3)$ & 3. $(0.2)$ & $3.7(0.3)$ & $2.7(0.2)$ & $3.2(0.2)$ & $4.2(0.3)$ \\
\hline He II 4686 & $8.1(0.6)$ & $9.8(0.7)$ & & $24(2)$ & & $21(1)$ & $16(1)$ & \\
\hline [Ar IV] $4711^{a}$ & & & & 4. $(0.3)$ & & $3.4(0.3)$ & $2.9(0.2)$ & \\
\hline [Ar IV] 4740 & & & & $3.2(0.2)$ & & $2.6(0.2)$ & $2.4(0.2)$ & \\
\hline $\mathrm{H} \beta$ & 100 & 100 & 100. & 100 & 100 & 100 & 100 & 100. \\
\hline [O III] 4959 & $157(11)$ & $155(11)$ & $191(14)$ & $224(16)$ & $200(14)$ & 194 (14) & $229(16)$ & $194(14)$ \\
\hline [O III] 5007 & $471(34)$ & $464(33)$ & $566(40)$ & $670(48)$ & 597 (43) & $583(42)$ & $685(49)$ & $573(41)$ \\
\hline He II 5412 & & & & $1.7(0.1)$ & & $1.6(0.1)$ & $1.2(0.1)$ & \\
\hline He I 5876 & $12(1)$ & $12(1)$ & $13(1)$ & $8.5(0.7)$ & $11(1)$ & $8.4(0.7)$ & 9. (0.1) & $11(1)$ \\
\hline [O I] 6300 & & $4.4(0.4)$ & $7.1(0.7)$ & & $3.5(0.3)$ & $1.7(0.2)$ & $2.7(0.3)$ & $6.3(0.6)$ \\
\hline [S III] 6312 & & $1.7(0.2)$ & $2.2(0.2)$ & $1.8(0.2)$ & 2. (0.2) & $1.7(0.2)$ & $1.9(0.2)$ & $2.1(0.2)$ \\
\hline [O I] 6363 & & $1.6(0.2)$ & $2.2(0.2)$ & & 1. (0.1) & & $0.8(0.2)$ & $1.9(0.2)$ \\
\hline [N II] 6548 & $7.2(0.7)$ & $7.9(0.8)$ & $8.9(0.9)$ & 1. (0.1) & $1.9(0.2)$ & $1.6(0.2)$ & $1.4(0.1)$ & $3.8(0.4)$ \\
\hline $\mathrm{H} \alpha^{b}$ & $282(28)$ & $282(28)$ & $286(29)$ & $279(28)$ & $282(28)$ & 279 (28) & 279 (28) & $279(28)$ \\
\hline [N II] 6583 & $16(2)$ & $24(2)$ & $28(3)$ & $2.3(0.2)$ & $5.6(0.6)$ & $3.6(0.4)$ & $4.2(0.4)$ & $11(1)$ \\
\hline He I 6678 & $2.7(0.3)$ & $3.3(0.3)$ & $3.7(0.4)$ & $2.5(0.3)$ & 3. $(0.3)$ & $2.6(0.3)$ & $2.7(0.3)$ & $3.1(3)$ \\
\hline [S II] 6716 & $13(1)$ & $29(3)$ & $36(4)$ & $4.1(0.4)$ & $13(1)$ & $7.6(0.8)$ & $9.2(1)$. & $22(2)$ \\
\hline [S II] 6731 & $9.0(0.9)$ & $20(2)$ & $25(3)$ & $2.8(0.3)$ & $9.2(1)$. & $5.4(0.6)$ & $6.4(0.7)$ & $16(2)$ \\
\hline$F(\mathrm{H} \beta)\left(10^{-14} \mathrm{erg} \mathrm{cm}^{-2} \mathrm{~s}^{-1}\right)$ & 0.5 & 1.4 & 4.6 & 5.8 & 5.0 & 2.3 & 7.9 & 5.1 \\
\hline$A_{\mathrm{V}}(\mathrm{mag})$ & 0.44 & 0.44 & 0.49 & 0.29 & 0.39 & 0.30 & 0.40 & 0.47 \\
\hline$T_{\mathrm{e}}[\mathrm{O} \mathrm{III}](\mathrm{kK})$ & $12.5 \pm 0.5$ & $11.9 \pm 0.4$ & $11 . \pm 0.4$ & $15.5 \pm 0.7$ & $13.5 \pm 0.5$ & $14.8 \pm 0.7$ & $15.1 \pm 0.7$ & $14 . \pm 0.6$ \\
\hline$n_{\mathrm{e}}[\mathrm{S} \mathrm{II}]\left(\mathrm{cm}^{-3}\right)$ & $<210$ & $<210$ & $<230$ & $<160$ & $<260$ & $<250$ & $<210$ & $<230$ \\
\hline$n_{\mathrm{e}}[\operatorname{Ar} \mathrm{IV}]\left(\mathrm{cm}^{-3}\right)$ & & & & $<3100$ & & $<2700$ & $600-3600$ & \\
\hline $\mathrm{He}^{+} / \mathrm{H}^{+} \times 10^{2}$ & $8.3 \pm 0.6$ & $8.9 \pm 0.6$ & $9.2 \pm 0.5$ & $6.6 \pm 0.3$ & $8.1 \pm 0.4$ & $6.5 \pm 0.3$ & $7.1 \pm 0.4$ & $8.8 \pm 0.5$ \\
\hline $\mathrm{He}^{2+} / \mathrm{H}^{+} \times 10^{2}$ & $0.68 \pm 0.05$ & $0.82 \pm 0.06$ & & $2 . \pm 0.1$ & & $1.8 \pm 0.1$ & $1.4 \pm 0.1$ & \\
\hline$\rightarrow \mathrm{He} / \mathrm{H} \times 10^{2}$ & $9.0 \pm 0.6$ & $9.8 \pm 0.8$ & $9.2 \pm 0.5$ & $8.6 \pm 0.1$ & $8.1 \pm 0.4$ & $8.3 \pm 0.4$ & $8.5 \pm 0.4$ & $8.8 \pm 0.5$ \\
\hline $\mathrm{O}^{0+} / \mathrm{H}^{+} \times 10^{6}$ & & $4.9 \pm 0.3$ & $9.5 \pm 0.6$ & & $2.3 \pm 0.2$ & & $1.3 \pm 0.2$ & $3.8 \pm 0.3$ \\
\hline $\mathrm{O}^{+} / \mathrm{H}^{+} \times 10^{5}$ & $2.8 \pm 0.3$ & $4.5 \pm 0.5$ & $6.6 \pm 0.7$ & $0.39 \pm 0.04$ & $1.4 \pm 0.2$ & $0.72 \pm 0.08$ & $0.83 \pm 0.09$ & $2.7 \pm 0.3$ \\
\hline $\mathrm{O}^{2+} / \mathrm{H}^{+} \times 10^{5}$ & $8.1 \pm 0.3$ & $9.3 \pm 0.4$ & $14.5 \pm 0.6$ & $6.5 \pm 0.3$ & $8.3 \pm 0.4$ & $6.4 \pm 0.3$ & $7.1 \pm 0.3$ & $7.3 \pm 0.3$ \\
\hline$\rightarrow \mathrm{O} / \mathrm{H} \times 10^{4}$ & $1.2 \pm 0.1$ & $1.6 \pm 0.1$ & $2.2 \pm 0.1$ & $0.89 \pm 0.04$ & $1 . \pm 0.04$ & $0.91 \pm 0.04$ & $0.96 \pm 0.04$ & $1 . \pm 0.1$ \\
\hline $\mathrm{N}^{+} / \mathrm{H}^{+} \times 10^{6}$ & $2.1 \pm 0.2$ & $3 . \pm 0.2$ & $4.2 \pm 0.3$ & $1.9 \pm 0.1$ & $5.4 \pm 0.4$ & $3.3 \pm 0.2$ & $3.2 \pm 0.2$ & $9.9 \pm 0.7$ \\
\hline$\left(\rightarrow \mathrm{N} / \mathrm{O} \times 10^{2}\right)^{c}$ & $7.7 \pm 1$ & $6.6 \pm 0.8$ & $6.3 \pm 0.8$ & $5 . \pm 0.6$ & $3.8 \pm 0.5$ & $4.6 \pm 0.6$ & $3.9 \pm 0.5$ & $3.7 \pm 0.5$ \\
\hline $\mathrm{S}^{+} / \mathrm{H}^{+} \times 10^{7}$ & $3.1 \pm 0.2$ & $7.6 \pm 0.6$ & $11.2 \pm 0.8$ & $0.64 \pm 0.05$ & $2.7 \pm 0.2$ & $1.3 \pm 0.1$ & $1.5 \pm 0.1$ & $4.2 \pm 0.2$ \\
\hline $\mathrm{S}^{2+} / \mathrm{H}^{+} \times 10^{6}$ & & $2 . \pm 0.2$ & $3.6 \pm 0.3$ & $0.92 \pm 0.09$ & $1.5 \pm 0.1$ & $0.96 \pm 0.09$ & $1 . \pm 0.1$ & $1.4 \pm 0.1$ \\
\hline $\mathrm{Ar}^{3+} / \mathrm{H}^{+} \times 10^{7}$ & & & & $2.2 \pm 0.1$ & & $2 . \pm 0.1$ & $1.7 \pm 0.1$ & \\
\hline $\mathrm{Ne}^{2+} / \mathrm{H}^{+} \times 10^{5}$ & $2 . \pm 0.2$ & $2.5 \pm 0.2$ & $4.3 \pm 0.4$ & $1.4 \pm 0.1$ & $2.1 \pm 0.2$ & $1.3 \pm 0.1$ & $1.5 \pm 0.2$ & $2 . \pm 0.2$ \\
\hline
\end{tabular}

${ }^{a}$ The small contamination due to He I 4713 was corrected using the strength of He I 5876 and the theoretical He I ratios from Benjamin et al. (1999).

${ }^{b}$ Contamination due to He II 6560 negligible.

${ }^{c}$ Assuming $N\left(\mathrm{~N}^{+}\right) / N\left(\mathrm{O}^{+}\right)=N(\mathrm{~N}) / N(\mathrm{O})$. 


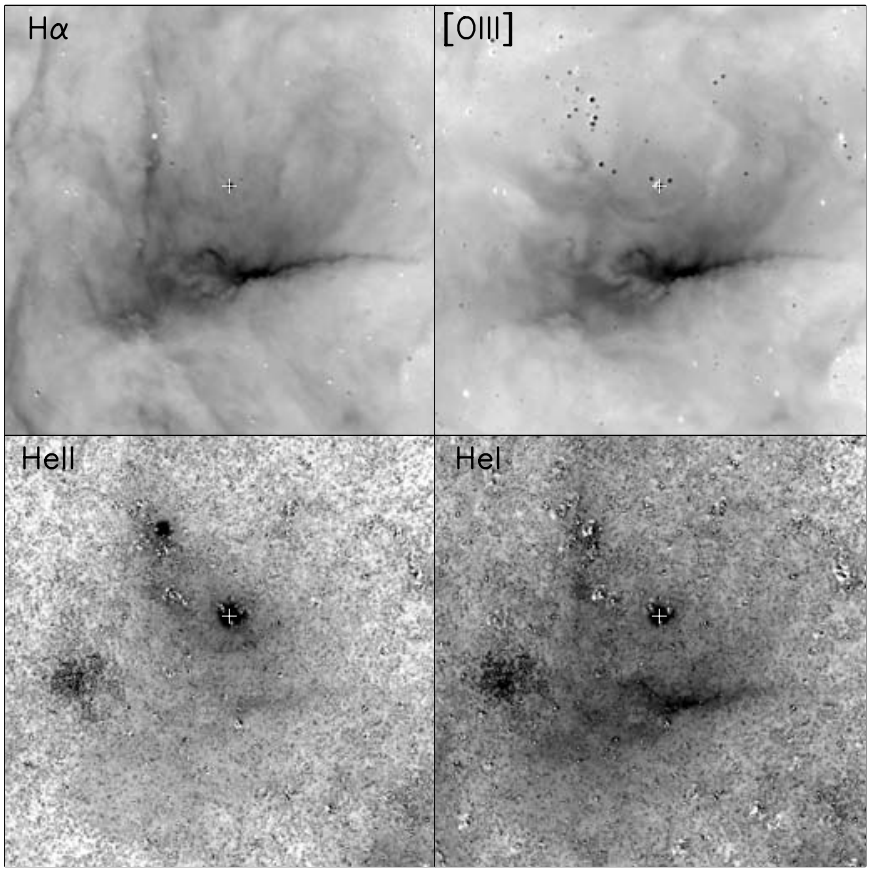

Fig. 3. FORS continuum subtracted $\mathrm{H} \alpha$, [O III], He II, and He I images of LMC BAT99-49 and its close surroundings. The images are $180^{\prime \prime} \times 180^{\prime \prime}$. A white cross indicates the WR star's position. The crowdiness of this field renders star-subtraction uttermost difficult. North is up and East to the left.

Spectra were extracted from three regions of interest for detailed analysis (Fig. 1): the first one samples the north of the star ( $11^{\prime \prime}$ long without the star); the second examines the close $15^{\prime \prime}$ south of the star and the third one explores the bright $\mathrm{H} \alpha$ region further south ( $38^{\prime \prime}$ long). None contains the WR star, nor another very bright star. The extinction-corrected line ratios and the physical properties derived for these regions are listed in Table 2. To get helium abundances, we used the emissivities at $12500 \mathrm{~K}$ computed by Benjamin et al. (1999) for He I and by Storey \& Hummer (1995) for H and He II. The presence of He II suggests that higher ionization states than $\mathrm{O}^{2+}$ exist as well, and we correct the $N(\mathrm{O}) / N(\mathrm{H})$ ratio like we did in Paper I. Compared to the LMC abundances (Russell \& Dopita 1990, see Table 3), no sign of enrichment in helium can be detected for the He II nebula. But while the oxygen abundance of the bright $\mathrm{H} \alpha$ region appears consistent with the LMC, the abundance closer to the star is slightly lower, and the N/O ratio higher than the LMC value. The close neighbourhood of the star appears thus enriched by a stellar wind displaying CNO processed material.

\subsection{Star $S M C A B 7$ and its associated nebula SMC N76}

N76 is a large circular nebula some 130 " in radius situated to the north of the SMC. Nebular He II emission in N76 was first discovered serendipitously by Tuohy \& Dopita (1983), while studying the neighbouring SNR 1E0101.2-7218. It was rediscovered later by Pakull \& Motch (1989b) who noted that the center of gravity of the He II emission coincides well with the
Table 3. Average abundances in the Magellanic Clouds, from Russel \& Dopita (1990).

\begin{tabular}{cccccc}
\hline \hline & $\mathrm{He} / \mathrm{H}$ & $\mathrm{O} / \mathrm{H}$ & $\mathrm{N} / \mathrm{H}$ & $\mathrm{N} / \mathrm{O}$ & $\mathrm{S} / \mathrm{H}$ \\
\hline $\mathrm{LMC}$ & 0.091 & $2.3 \times 10^{-4}$ & $1.2 \times 10^{-5}$ & 0.050 & $7.4 \times 10^{-6}$ \\
$\mathrm{SMC}$ & 0.083 & $1.3 \times 10^{-4}$ & $3.5 \times 10^{-6}$ & 0.026 & $6.8 \times 10^{-6}$ \\
\hline
\end{tabular}

WR star AB7. Spectra taken by Niemela et al. (1991) show a He II region of size 144", that fills a "central hole seen in He I and $\mathrm{H} \alpha$ ". Because of the presence of the He II nebula, this star, like BAT99-2, was classified as WN1 by Pakull (1991). More recent studies of the star undertaken by Niemela et al. (2002) and by Foellmi et al. (2003a) led to a re-classification of the star as $\mathrm{WN} 2+\mathrm{O} 6 \mathrm{I}(\mathrm{f})$ and $\mathrm{WN} 4+\mathrm{O} 6 \mathrm{I}(\mathrm{f})$, respectively.

Figure 2 shows an $\mathrm{H} \alpha$ image of the whole field. N76 nearly completely fills the FORS CCD. Its surface brightness is not totally uniform: the nebula brightens to the east, most probably indicating a density gradient. No "central hole" is readily apparent, but many filamentary features cover the whole nebula. N76 also presents pillar-like features some 92" west of $\mathrm{AB} 7$, a quite rare type of structures, suggesting that the nebula is situated on the edge of a molecular cloud being photoevaporated. These structures may hide a second generation of stars (Walborn 2002). We also note the presence, to the southeast of $\mathrm{AB} 7$, of a bright $\mathrm{H} \alpha$ knot of diameter 16", N76A or DEM S123, which we will discuss more below.

The He I emission appears well correlated with $\mathrm{H} \alpha$, whilst the [O III] image shows several differences: mainly, the SNR is clearly visible to the northeast of the field, and N76 is slightly smaller in this line (124" radius). Some of the filamentary features, the pillars and the bright $\mathrm{H} \alpha$ emitter DEM S123 present a lower $[\mathrm{O} \mathrm{III}] / \mathrm{H} \alpha$ ratio than its surroundings. On the other hand, the He II image shows a bright elliptic nebula of size $128^{\prime \prime} \times 146^{\prime \prime}$. Like in $\mathrm{H} \alpha$, the surface brightness is not uniform and the nebula appears composed of intrincate filaments making a reversed "S". These filaments also appears in $\mathrm{H} \alpha$, but they are rather faint. More extended, fainter He II emission is also detectable, corresponding more or less in size with the $\mathrm{H} \alpha$ nebula.

Garnett et al. (1991b) found an average I(He II $\lambda 4686) /$ $I(\mathrm{H} \beta)$ of 0.13 at their slit position, but we find that this ratio can actually reach higher values locally (see below). Assuming a spherical geometry, the same authors derived a luminosity $L(\mathrm{H} \beta)$ of $1.1 \times 10^{38} \mathrm{erg} \mathrm{s}^{-1}$ and $L(\mathrm{He}$ II $\lambda 4686)=5.7 \times 10^{36} \mathrm{erg} \mathrm{s}^{-1}$ for a distance of $78 \mathrm{kpc}$, or $L(\mathrm{H} \beta)=6.2 \times 10^{37} \mathrm{erg} \mathrm{s}^{-1}$ and $L(\mathrm{He}$ II $\lambda 4686)=3.2 \times$ $10^{36} \mathrm{erg} \mathrm{s}^{-1}$ for our adopted SMC distance of $59 \mathrm{kpc}$. Another estimate was made by Pakull \& Bianchi (1991), apparently using spectrophotometry and the same approximations as Garnett et al. (1991b). They found a flux in He II $\lambda 4686$ of $2.5 \times 10^{-12} \mathrm{erg} \mathrm{cm}^{-2} \mathrm{~s}^{-1}$, or $L(\mathrm{He}$ II $\lambda 4686)=1 \times 10^{36} \mathrm{erg} \mathrm{s}^{-1}$ for a distance of $59 \mathrm{kpc}$. Using our images calibrated with our FORS spectrophotometry, we find a $\mathrm{H} \beta$ luminosity, excluding DEM S123, of $3.0 \times 10^{37} \mathrm{erg} \mathrm{s}^{-1}$ inside a circular aperture of $130^{\prime \prime}$ in radius and a He II $\lambda 4686$ luminosity of $4.5 \times 10^{36} \mathrm{erg} \mathrm{s}^{-1}$ inside an elliptic aperture of $120^{\prime \prime} \times 152^{\prime \prime}$, 


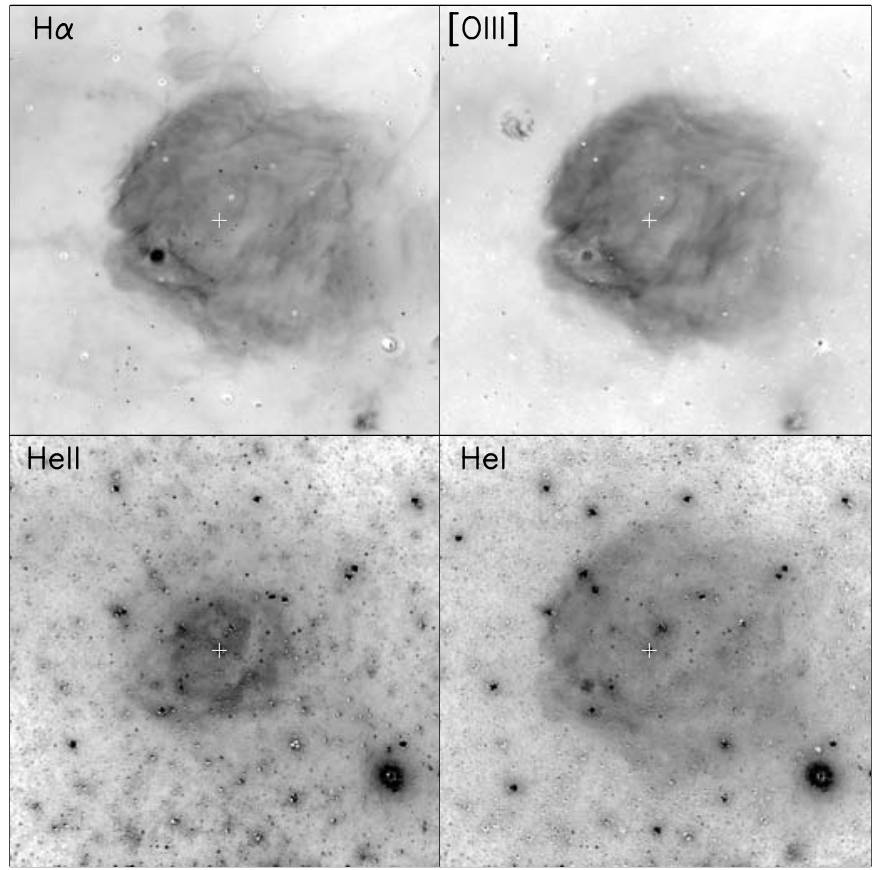

Fig. 4. FORS continuum subtracted $\mathrm{H} \alpha$, [O III], He II, and $\mathrm{He}$ I images of SMC N76. The images are $394^{\prime \prime} \times 394^{\prime \prime}$. A white cross indicates the position of SMC AB7 (note that the star is wrongly identified in Dopita et al. 1994). The SNR is the feature to the NE of the field only visible in [O III]. North is up and East to the left.

after a reddening correction of $A_{\mathrm{V}}=0.3 \mathrm{mag}$ and for a distance of $59 \mathrm{kpc}$. Since a fainter halo is present, we estimate that the He II $\lambda 4686$ flux may still be underestimated by $10 \%$. Our He II fluxes are larger than those obtained in the previous studies, but we use the actual surface brightness' distribution of the whole nebula, not an extrapolation of a one-position measurement. Our measured $\mathrm{H} \beta$ and $\mathrm{He}$ II $\lambda 4686$ fluxes correspond to ionizing fluxes $Q\left(H_{0}\right)=6.5 \times 10^{49}$ photons s $^{-1}$ and $Q\left(\mathrm{He}^{+}\right)=5.1 \times 10^{48}$ photons s ${ }^{-1}$. In the models of Smith et al. (2002), these values indicate a WN star hotter than their hottest model, of temperature $120 \mathrm{kK}$ (for $Z=0.05-0.2 Z_{\odot}$, $Q\left(\mathrm{He}^{+}\right)=3-4 \times 10^{48}$ photons s$\left.{ }^{-1}\right)$. We know that some $\mathrm{O}$ stars within N76 could contribute to the ionization of the $\mathrm{H}$ nebula (the companion of AB7, an O6If star; AzV 327, an O9.7Iab star; [MWD2000] h53-91 and 137 (Massey et al. 2000), O8.5 V and III stars, respectively), but according to the same models, the O-type stars certainly do not account for the $\mathrm{He}^{+}$ionization: at most, these stars would contribute by $8.5 \times 10^{49} \mathrm{H}$ ionizing photons $\mathrm{s}^{-1}$ and $3 \times 10^{44} \mathrm{He}^{+}$ionizing photons s ${ }^{-1}$. The He II nebula remains a puzzle: either AB7 is an ultra-hot WN star, or the models need to be adapted or revised, or another source of He II ionization is present. In this respect, we note that AB7 does not appear as a particularly bright X-ray source (Foellmi et al. 2003a), casting doubts on an X-ray ionization contribution. Another possibility to produce a high excitation nebula requires high velocity shocks (Garnett et al. 1991b). However, so far no kinematic study of N76 was undertaken, and we thus ignore whether any high-velocity motions are present. High dispersion spectroscopy of N76 would thus be valuable in order to better understand this peculiar object.

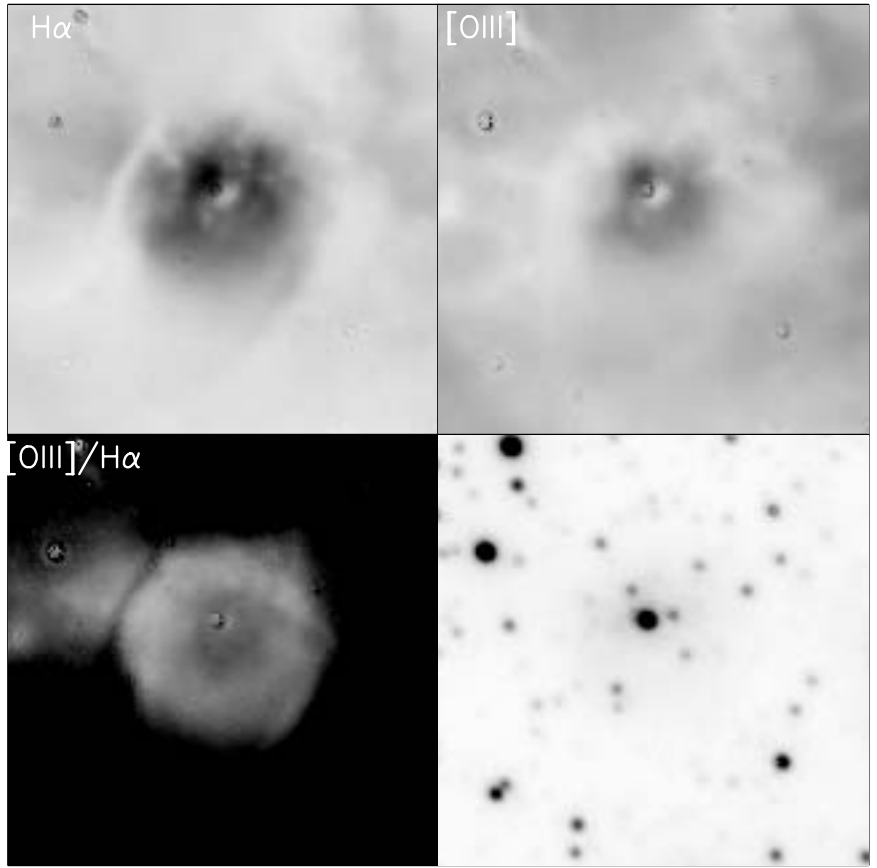

Fig. 5. Upper part: FORS continuum subtracted $\mathrm{H} \alpha$ and [O III] images of SMC N76A (or DEM S 123). Bottom part: [O III]/H $\alpha$ ratio and image taken with the continuum filter centered on $6665 \AA$. The images are $32^{\prime \prime} \times 32^{\prime \prime}$. North is up and East to the left. Contrary to the other figures of this paper, the greyscale is a linear scale, not a square root scale.

We select spectra from five regions, avoiding the brightest stars, for further detailed investigation (Fig. 2): three cover the central He II emission region (W1, 49" long; E1, 14" long; and E2, 38" long), while the other two explore the outer parts of the nebula where He II is barely visible but not measurable (W2, 78" long and E3, 36" long). The extinction-corrected line ratios for these regions are listed in Table 2. In the same way as for BAT99-49, we then derived temperatures, densities and abundances in these regions. No significant enrichment in helium can be detected for the He II nebula but the oxygen abundance is at least 35\% lower, and the N/O ratio is at least $40 \%$ higher than the SMC's average values (Russell \& Dopita 1990, see Table 3). Like in BAT99-2 and 49, this suggests a small enrichment by stellar winds, but in the case of N76, the nebula over which this enrichment is seen is much larger.

\subsubsection{LMC N76A (or DEM S 123)}

To the southeast of AB7 is situated N76 A (or DEM S 123, see Fig. 5), a bright $\mathrm{H} \alpha$ knot of radius $\sim 8^{\prime \prime}$ similar to compact $\mathrm{H}$ II regions such as N11A in the LMC (Heydari-Malayeri et al. 2001). Following Heydari-Malayeri et al. (2001 and references therein), these compact regions are created by massive stars just leaving their natal molecular cloud. The same authors also called this type of objects "High Excitation Blobs" (HEBs). In N76A, one very bright star lies near the center of this nebula, and five others are situated closer to its periphery (see Fig. 5). Unfortunately, the spectral types of these stars are unknown and may be worth further investigation. The brightest region 


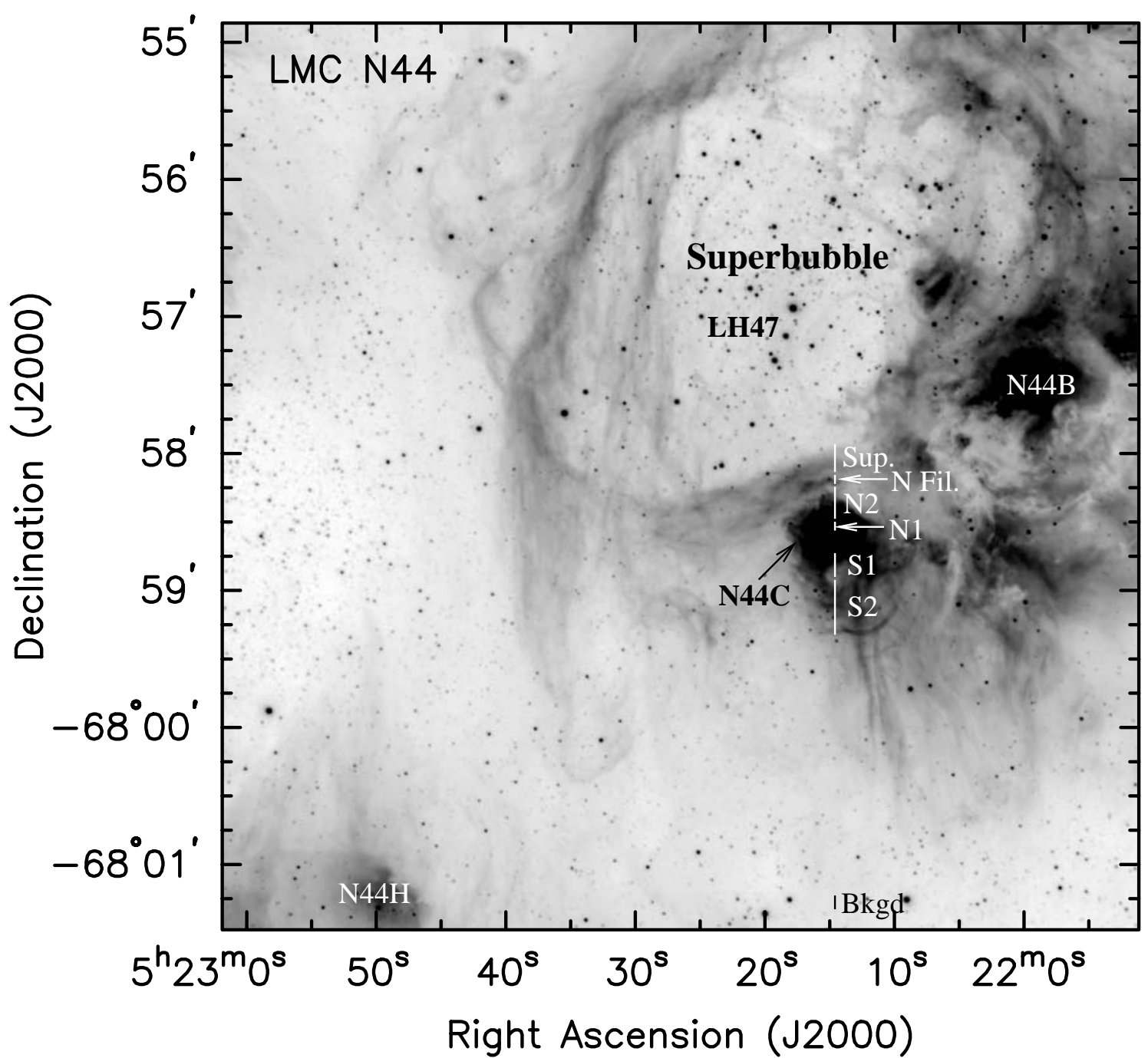

Fig. 6. FORS H $\alpha$ image of LMC N44. The different regions used for spectral analysis are marked by a solid line. Features discussed in the text are labelled.

of N76A is situated just to the NE of the brightest star, whilst a region of suppressed brightness is directly symetrical to it with respect of the star. A diffuse envelope with few remarkable features surrounds the center of this nebula, and a dust lane borders it to the east.

As mentioned above, the $[\mathrm{OIII}] / \mathrm{H} \alpha$ ratio of $\mathrm{N} 76 \mathrm{~A}$ is much lower than that of its direct surroundings. It is actually the lowest to the northern and eastern edges of the nebula $([\mathrm{O}$ III $] / \mathrm{H} \alpha \sim 0.3)$, while it peaks to the southwest of the brightest star $([\mathrm{O} \mathrm{III}] / \mathrm{H} \alpha \sim 0.7)$. In a similar way as above, we estimate the $\mathrm{H} \alpha$ and [O III] fluxes in an aperture of 8 " radius and find an observed $L(\mathrm{H} \alpha) \sim 1 \times 10^{36} \mathrm{erg} \mathrm{s}^{-1}$ and an observed $L([\mathrm{O} \mathrm{III}] \lambda 5007) \sim 6 \times 10^{35} \mathrm{erg} \mathrm{s}^{-1}$ for a distance of $59 \mathrm{kpc}$. However, since the reddening of this region is not known, we thus cannot derive the actual luminosities. But the observed $\mathrm{H} \alpha$ luminosity can still be used to estimate the minimum requirement on the spectral type in the case of a unique ionizing star: it corresponds to an ionizing flux of $\sim 7 \times 10^{47}$ ionizing photons $\mathrm{s}^{-1}$, which can be explained by an O9V type star. Further investigations should enable to find the reddening of the nebula and the exact nature of its embedded stars, but also to check the predicted correlation between the $[\mathrm{O} \mathrm{III}] / \mathrm{H} \beta$ ratio and the $\mathrm{H} \beta$ flux for the HEBs (Heydari-Malayeri et al. 2001).

\section{3. $L M C N 44 C$}

$\mathrm{N} 44 \mathrm{C}$ is a bright elongated $\mathrm{H} \alpha$ nebula some $53^{\prime \prime} \times 65^{\prime \prime}$ situated to the southwest of a superbubble blown by the OB association LH47 (see Fig. 6). Nebular He II $\lambda 4686$ emission was detected in N44C by Stasińska et al. (1986) who also provided the first spectra of this nebula. Examining a high dispersion $\mathrm{H} \alpha$ spectrum of N44C, Goudis \& Meaburn (1984) claimed to have discovered gas moving at $-120 \mathrm{~km} \mathrm{~s}^{-1}$ in this nebula, but it was found later that this component was actually "only" the He II $\lambda 6560$ line (Garnett et al. 2000). Two bright stars in the center of N44C have been detected by Stasińska et al. (1986) and labelled Star \#2 for the northern one and Star \#1 for the southern one. Star \#2 is a rather hot O-type star while Star \#1 is a cool G-K foreground star (Stasińska et al. 1986; Garnett et al. 2000).

In the absence of high velocity motions or any very hot star, it was suggested that the $\mathrm{He}^{+}$ionization results from an 


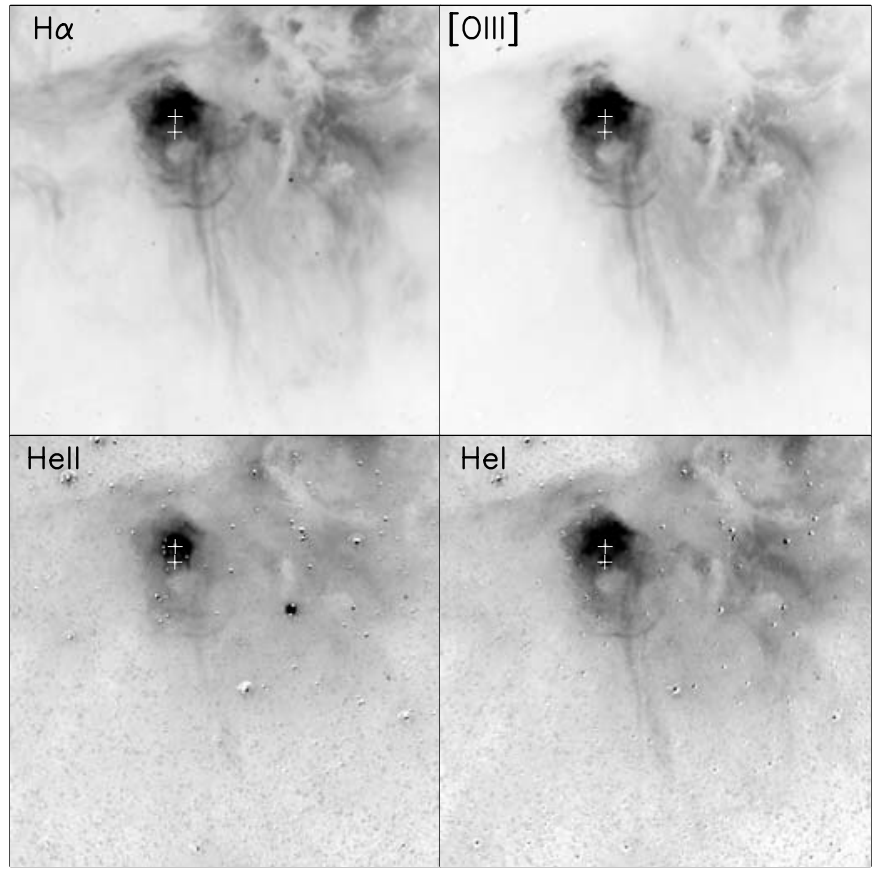

Fig. 7. FORS continuum subtracted $\mathrm{H} \alpha$, [O III], He II, and He I images of LMC N44C and its close surroundings. The images are $200^{\prime \prime} \times 200^{\prime \prime}$. The upper white cross indicates the Star \# 2 of Stasińska et al. (1986), and the lower one shows the position of Star \# 1. North is up and East to the left.

intense X-ray source inside the nebula. Such a process is actually seen at work around another object of the LMC, LMC X-1, and in this case, the He II nebula is complex and very extended. If the X-ray source is transient, the high excitation may last for some time after the X-ray source has switched off. For N44C, the He II nebula has been proposed to be a fossil X-ray nebula corresponding to the once recorded X-ray transient LMC X-5 (Pakull \& Motch 1989a). An extensive study of N44C was recently presented by Garnett et al. (2000) who evaluated that $\mathrm{He}$ II is currently recombining with a characteristic timescale of 20 yr. Since their spectrum was taken in 1991 and Stasińska's in 1985, it was obviously interesting to monitor the evolution of the He II emission ten to fifteen years later.

Images of $\mathrm{N} 44 \mathrm{C}$ in the four nebular filters are presented in Fig. 7. The $\mathrm{H} \alpha$ and [O III] images have been described in length by Garnett et al. (2000) and we will not repeat the discussion here. The He I image correlates perfectly with $\mathrm{H} \alpha$, except for a slightly He I depleted region where the He II emission is the brightest. The He II image presents a very bright arc north to Star \#2 and a fainter, more extended emission. A close-up on the arc, whose radius is $\sim 6.5^{\prime \prime}$, is presented in Fig. 8. It seems to closely envelope the two stars. This arc does not correspond to any feature in the other nebular lines. On the contrary, the halo is as extended as the $\mathrm{H} \alpha$ nebula, and well correlated with it. However, an important part of this halo is actually made of [Ar IV] emission: in the spectra, the observed [Ar IV] $\lambda 4711+$ He I $\lambda 4713$ blend can reach four times the intensity of the He II $\lambda 4686$ line. In the rest of the field, emission in the He II filter is spotted in N44B, N44H and in the superbubble. However, these features are most probably not due to

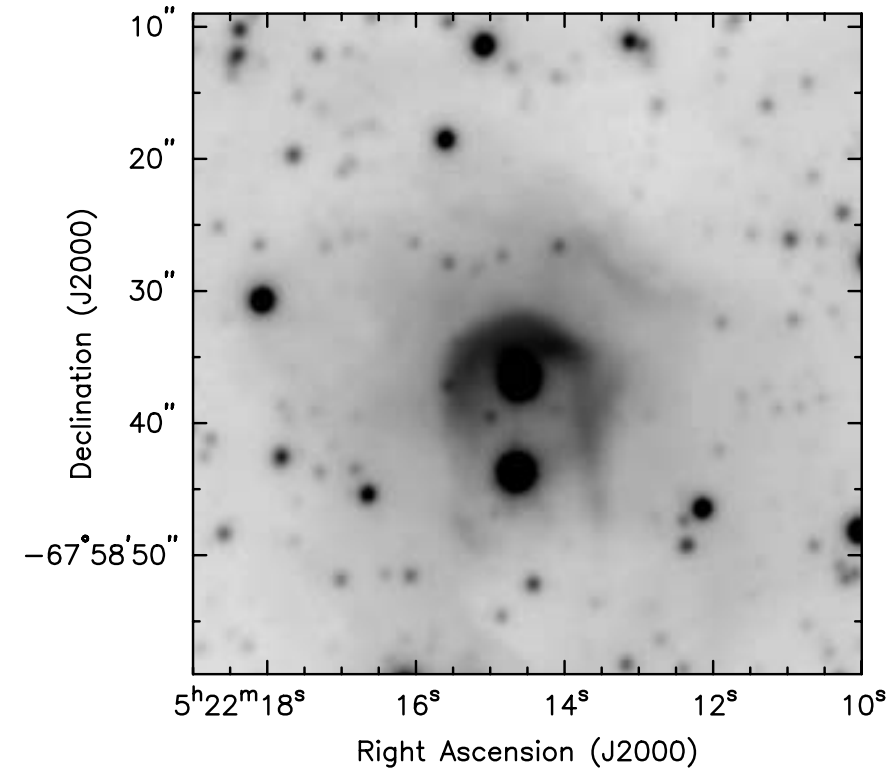

Fig. 8. Close-up on LMC N44C in the He II $\lambda 4686$ line (the image is not continuum subtracted). Stars \# 1 and 2 are clearly visible, as is a bright arc-like He II emission region enveloping the Star \# 2. Fainter, more extended emission also exists, but is more apparent in Fig. 7.

a nebular He II emission. In fact, our spectrum reveals a faint [Fe III] $\lambda 4658$ line in the superbubble and Dufour (1975) detected [Fe III] $\lambda 4658$ in N44B, although Stasińska et al. (1986) think that Dufour actually observed N44C, not B, and mistook He II $\lambda 4686$ for the [Fe III] line. Unfortunately, we do not have any information in the case of N44H's emission, since our spectra do not cover this region.

We choose six regions to further examine the physical properties of the nebula and its surroundings (see Fig. 6): the He II arc situated directly north of Star \#2 (N1, 4.6" long), the northern part of N44C (N2, 15" long), the region directly south of Star \#1 (S1, 11" long), the southern part of N44C that seems composed of interlaced arcs (S2, 25" long), a filament just north of N44C which possesses an ionization closer to N44C than to the superbubble (N Fil., 5.2" long), and finally the superbubble itself (Sup., 13" long). The dereddened line ratios of these six regions, together with the derived physical properties, are presented in Table 4 . The helium abundance is close to normal in all cases, except for the superbubble where it is rather low. The oxygen abundance is at most slightly smaller than normal, but the N/O ratio always reflects the LMC average (see Table 3). No significant chemical enrichment is thus detected for this nebula.

We estimate the $\mathrm{H} \alpha$ and He II fluxes by calibrating the corresponding images with our spectrophotometry. For the whole $\mathrm{H} \alpha$ nebula $\left(53^{\prime \prime} \times 65^{\prime \prime}\right)$, we derived a $\mathrm{H} \beta$ luminosity of $9 \times 10^{36} \mathrm{erg} \mathrm{s}^{-1}$ after a reddening correction $A_{\mathrm{V}}=0.55 \mathrm{mag}$ and for a distance of $50 \mathrm{kpc}$. Note that the $\mathrm{H} \beta$ luminosity given in Garnett et al. (2000) is an order of magnitude larger than this value. However, this is most probably a typographical error: Garnett et al. (2000) quote a reddeningcorrected $\mathrm{H} \beta$ flux of $3 \times 10^{-10} \mathrm{erg} \mathrm{cm}^{-2} \mathrm{~s}^{-1}$ for the sole N44C, while Caplan \& Deharveng (1985) give an observed 
Table 4. Same as Table 2 for LMC N44C.

\begin{tabular}{|c|c|c|c|c|c|c|}
\hline & N1 & $\mathrm{N} 2$ & S1 & S2 & N Fil. & Sup. \\
\hline [Fe II $] 3712$ & & & & & & $9.1(1)$. \\
\hline [O II] 3727 & $91(9)$ & $220(23)$ & $199(21)$ & 353 (37) & $547(57)$ & $486(51)$ \\
\hline $\mathrm{H} 12$ & 4. $(0.4)$ & $3.7(0.4)$ & $4.7(0.5)$ & $4.9(0.5)$ & $4.6(0.5)$ & $5.6(0.6)$ \\
\hline H11 & $4.8(0.5)$ & $4.6(0.5)$ & $6.2(0.6)$ & $5.9(0.6)$ & $6.3(0.7)$ & $6.4(0.7)$ \\
\hline $\mathrm{H} 10$ & $5.9(0.6)$ & $5.8(0.6)$ & $7.4(0.8)$ & 7. $(0.7)$ & $6.8(0.7)$ & $6.1(0.6)$ \\
\hline H9 & $7.5(0.8)$ & $7.8(0.8)$ & $8.8(0.9)$ & 9. $(0.9)$ & $8.3(0.8)$ & $8.1(0.8)$ \\
\hline [Ne III] 3868 & $63(6)$ & $73(7)$ & $59(6)$ & $56(6)$ & $65(6)$ & $11(1)$ \\
\hline $\mathrm{H} 8+\mathrm{He} \mathrm{I}$ & $19(2)$ & $21(2)$ & $20(2)$ & $20(2)$ & $19(2)$ & $20(2)$ \\
\hline$[\mathrm{Ne} \mathrm{III}]+\mathrm{H} \epsilon$ & $35(3)$ & $39(4)$ & $34(3)$ & $35(3)$ & $35(3)$ & $22(2)$ \\
\hline He I 4026 & $2.1(0.2)$ & $2.2(0.2)$ & $1.7(0.2)$ & $1.7(0.2)$ & $1.8(0.2)$ & 1.3: \\
\hline [S II] 4068 & $1.3(0.1)$ & $2.7(0.3)$ & $2.4:$ & 4. $(0.4)$ & $6.7(0.6)$ & 4.3: \\
\hline [S II] 4076 & $0.4(0.2)$ & $0.5(0.2)$ & & $0.6(0.1)$ & 1. $(0.2)$ & \\
\hline $\mathrm{H} \delta$ & $29(3)$ & $29(3)$ & $30(3)$ & $30(3)$ & $29(3)$ & $29(3)$ \\
\hline $\mathrm{H} \gamma$ & $50(4)$ & $50(4)$ & $50(4)$ & $50(4)$ & $50(4)$ & $50(4)$ \\
\hline [O III] 4363 & $7.4(0.6)$ & $7.3(0.6)$ & $6.7(0.5)$ & $4.9(0.4)$ & $5.5(0.4)$ & $0.8(0.2)$ \\
\hline He I 4389 & $0.4(0.2)$ & $0.5(0.3)$ & & & & \\
\hline He I 4471 & $3.6(0.3)$ & $4.4(0.3)$ & $3.8(0.3)$ & $3.7(0.3)$ & $4.1(0.3)$ & $3.6(0.3)$ \\
\hline He II 4542 & $0.4(0.2)$ & & & & & \\
\hline He II 4686 & $14(0.1)$ & $0.45(0.03)$ & $4.3(0.3)$ & $1.7(0.1)$ & & \\
\hline [Ar IV] $4711^{a}$ & $3.3(0.3)$ & $1.2(0.1)$ & $1.4(0.1)$ & & & \\
\hline [Ar IV] 4740 & $2.5(0.2)$ & 1. $(0.2)$ & $1.2(0.1)$ & & & \\
\hline $\mathrm{H} \beta$ & 100. & 100 & 100 & 100 & 100. & 100. \\
\hline He I 4922 & $0.9(0.2)$ & $1.1(0.1)$ & $1.1(0.1)$ & 1. $(0.2)$ & $1.1(0.1)$ & 1.6: \\
\hline [O III] 4959 & 242 (17) & $250(18)$ & $217(15)$ & $178(13)$ & $161(11)$ & $36(3)$ \\
\hline [Fe III] 4986 & & & & & & $2.2(0.2)$ \\
\hline [O III] 5007 & $719(51)$ & 747 (53) & $649(46)$ & $534(38)$ & $478(34)$ & $108(8)$ \\
\hline [N I] 5198+5200 & $0.2(0.1)$ & $0.6(0.1)$ & & 1. $(0.2)$ & 2. $(0.2)$ & $1 .:$ \\
\hline He II 5412 & $1.1(0.1)$ & & $0.7(0.1)$ & & & \\
\hline [Cl III] 5517 & $0.4(0.2)$ & $0.5(0.1)$ & $0.6(0.1)$ & 0.6: & & \\
\hline [Cl III] 5538 & $0.3(0.1)$ & $0.4(0.2)$ & $0.5(0.1)$ & $0.5:$ & & \\
\hline [N II] 5755 & $0.13(0.07)$ & $0.3(0.2)$ & & $0.5(0.1)$ & $0.7(0.1)$ & \\
\hline He I 5876 & $10(1)$ & $12(1)$ & $10(1)$ & $10(1)$ & $9.8(0.8)$ & $9.3(0.1)$ \\
\hline [Fe III] 6096 & & & & & & $1.5(0.1)$ \\
\hline [O I] 6300 & $1.9(0.2)$ & $6.8(0.7)$ & $3.9(0.4)$ & $8.4(0.8)$ & $14(1)$ & $4.1(0.4)$ \\
\hline [S III] 6312 & $1.4(0.1)$ & $1.9(0.2)$ & $1.6(0.2)$ & $1.9(0.2)$ & 2. $(0.2)$ & 1. (0.1) \\
\hline [O I] 6363 & $0.8(0.2)$ & $2.4(0.2)$ & $1.4(0.1)$ & $2.9(0.3)$ & $4.8(0.5)$ & $1.8(0.2)$ \\
\hline [N II] 6548 & 2. $(0.2)$ & $5.2(0.5)$ & 5. $(0.5)$ & $8.7(0.9)$ & $14(1)$ & $13(1)$ \\
\hline $\mathrm{H} \alpha^{a}$ & $282(28)$ & $282(28)$ & $282(28)$ & $282(28)$ & $282(28)$ & $286(29)$ \\
\hline [N II] 6583 & $6.5(0.7)$ & $16(2)$ & $16(2)$ & $27(3)$ & $42(4)$ & $38(4)$ \\
\hline He I 6678 & $2.9(0.3)$ & $3.3(0.3)$ & $2.9(0.3)$ & $2.7(0.3)$ & $2.8(0.3)$ & $2.6(0.3)$ \\
\hline [S II] 6716 & $8.9(0.9)$ & $22(2)$ & $24(3)$ & $42(4)$ & $58(6)$ & $51(5)$ \\
\hline [S II] 6731 & 7. $(0.7)$ & $17(2)$ & $17(2)$ & $30(3)$ & $42(4)$ & $36(4)$ \\
\hline$F(\mathrm{H} \beta)\left(10^{-14} \mathrm{erg} \mathrm{cm}^{-2} \mathrm{~s}^{-1}\right)$ & 18 & 23 & 8.4 & 13 & 1.7 & 2.4 \\
\hline$A_{\mathrm{V}}(\mathrm{mag})$ & 0.58 & 0.60 & 0.52 & 0.53 & 0.48 & 0.43 \\
\hline$T_{\mathrm{e}}[\mathrm{O} \mathrm{III}](\mathrm{kK})$ & $11.7 \pm 0.4$ & $11.5 \pm 0.4$ & $11.7 \pm 0.4$ & $11.3 \pm 0.4$ & $12.2 \pm 0.4$ & $10.5 \pm 0.9$ \\
\hline$T_{\mathrm{e}}[\mathrm{S} \mathrm{II}](\mathrm{kK})$ & $11.8 \pm 2$ & $9.9 \pm 1$ & & $8.3 \pm 0.4$ & $9.3 \pm 0.8$ & \\
\hline$T_{\mathrm{e}}[\mathrm{N}$ II $](\mathrm{kK})$ & $12 \pm 5$ & $12 \pm 4$ & & $11.4 \pm 1.4$ & $10.7 \pm 1.1$ & \\
\hline$n_{\mathrm{e}}[\mathrm{S} \mathrm{II}]\left(\mathrm{cm}^{-3}\right)$ & $<420$ & $<410$ & $<260$ & $<250$ & $<270$ & $<240$ \\
\hline$n_{\mathrm{e}}[\mathrm{Ar} \mathrm{IV}]\left(\mathrm{cm}^{-3}\right)$ & $<2400$ & $<5400$ & $600-3600$ & & & \\
\hline
\end{tabular}


Table 4. continued.

\begin{tabular}{lcccccc}
\hline \hline & $\mathrm{N} 1$ & $\mathrm{~N} 2$ & $\mathrm{~S} 1$ & $\mathrm{~S} 2$ & $\mathrm{~N}$ Fil. & Sup. \\
\hline$\left(\mathrm{He}^{+} / \mathrm{H}^{+} \times 10^{2}\right)^{b}$ & $7.7 \pm 0.5$ & $9 . \pm 0.6$ & $7.9 \pm 0.3$ & $7.6 \pm 0.4$ & $7.9 \pm 0.3$ & $6.9 \pm 0.3$ \\
$\mathrm{He}^{2+} / \mathrm{H}^{+} \times 10^{2}$ & $1.2 \pm 0.2$ & $0.038 \pm 0.003$ & $0.55 \pm 0.08$ & $0.14 \pm 0.01$ & & \\
$\rightarrow \mathrm{He} / \mathrm{H} \times 10^{2}$ & $8.9 \pm 0.5$ & $9.1 \pm 0.6$ & $8.4 \pm 0.3$ & $7.7 \pm 0.4$ & $7.9 \pm 0.3$ & $6.9 \pm 0.3$ \\
$\mathrm{O}^{0+} / \mathrm{H}^{+} \times 10^{6}$ & $2.4 \pm 0.3$ & $8.3 \pm 0.6$ & $4.5 \pm 0.2$ & $11 \pm 1$ & $14 \pm 1$ & $7.9 \pm 0.5$ \\
$\mathrm{O}^{+} / \mathrm{H}^{+} \times 10^{5}$ & $1.8 \pm 0.2$ & $4.7 \pm 0.5$ & $4 . \pm 0.4$ & $8 . \pm 0.8$ & $9.3 \pm 1$. & $15 \pm 2$ \\
$\mathrm{O}^{2+} / \mathrm{H}^{+} \times 10^{4}$ & $1.5 \pm 0.1$ & $1.7 \pm 0.1$ & $1.4 \pm 0.1$ & $1.2 \pm 0.1$ & $0.89 \pm 0.04$ & $0.32 \pm 0.03$ \\
$\rightarrow \mathrm{O} / \mathrm{H} \times 10^{4}$ & $2 . \pm 0.1$ & $2.2 \pm 0.1$ & $1.9 \pm 0.1$ & $2.2 \pm 0.1$ & $2.0 \pm 0.1$ & $1.9 \pm 0.2$ \\
$\mathrm{~N}^{0+} / \mathrm{H}^{+} \times 10^{7}$ & $0.84 \pm 0.04$ & $2.3 \pm 0.5$ & & $3.8 \pm 0.8$ & $5.9 \pm 0.5$ & \\
$\mathrm{~N}^{+} / \mathrm{H}^{+} \times 10^{6}$ & $0.84 \pm 0.16$ & $2.2 \pm 0.4$ & $2 . \pm 0.1$ & $3.9 \pm 0.3$ & $4.6 \pm 0.4$ & $6.5 \pm 0.5$ \\
$\left(\rightarrow \mathrm{N} / \mathrm{O} \times 10^{2}\right)^{a}$ & $4.7 \pm 1$. & $4.8 \pm 0.9$ & $5.2 \pm 0.6$ & $5.3 \pm 0.7$ & $4.9 \pm 0.6$ & $4.4 \pm 0.6$ \\
$\left(\mathrm{~S}^{+} / \mathrm{H}^{+} \times 10^{7}\right)^{c}$ & $2.6 \pm 0.2$ & $5.9 \pm 0.4$ & $6.7 \pm 0.5$ & $10 \pm 1$ & $13 \pm 1$ & $16 \pm 1$ \\
$\mathrm{~S}^{2+} / \mathrm{H}^{+} \times 10^{6}$ & $1.8 \pm 0.2$ & $2.6 \pm 0.3$ & $2.1 \pm 0.2$ & $2.8 \pm 0.3$ & $2.2 \pm 0.2$ & $2 \pm 0.2$ \\
$\mathrm{Ar}^{3+} / \mathrm{H}^{+} \times 10^{7}$ & $3.4 \pm 0.2$ & $1.3 \pm 0.2$ & $1.3 \pm 0.1$ & & & \\
$\mathrm{Ne}^{2+} / \mathrm{H}^{+} \times 10^{5}$ & $3.8 \pm 0.4$ & $4.7 \pm 0.5$ & $3.6 \pm 0.4$ & $3.9 \pm 0.4$ & $3.4 \pm 0.3$ & $0.96 \pm 0.10$ \\
$\mathrm{Cl}^{2+} / \mathrm{H}^{+} \times 10^{8}$ & $2.4 \pm 0.9$ & $3.6 \pm 1$. & $4.5 \pm 0.7$ & & & \\
\hline
\end{tabular}

${ }^{a}$ Same remarks as in Table 2.

${ }^{b}$ To derive this abundance, the weigth of the fainter He I $\lambda$ 4026, 4389 and 4922 lines was reduced to 0.5 .

${ }^{c}$ To derive this abundance, the weigth of the $[\mathrm{S} \mathrm{II}] \lambda 4076$ line was reduced to 0.5 .

flux of $1.1 \times 10^{-10} \mathrm{erg} \mathrm{cm}^{-2} \mathrm{~s}^{-1}$ for $\mathrm{N} 44 \mathrm{~B}+\mathrm{C}(\mathrm{N} 44 \mathrm{~B}$ is more extended and as bright as $\mathrm{N} 44 \mathrm{C}$ !). The analysis of the HST images used by Garnett et al. (2000) and several other calibrated $\mathrm{H} \alpha$ images of this nebula confirm our flux estimate $^{3}$. Our $\mathrm{H} \beta$ luminosity corresponds to an ionizing flux of $1.9 \times 10^{49}$ photons $\mathrm{s}^{-1}$. Due to the pollution by the nebular lines and to possible abundance anomalies, the spectral type of Star \#2 is uncertain. Pakull \& Motch (1989a) proposed an O4-6 type, while Garnett et al. (2000) prefered ON7 III-V. The observed $Q\left(H_{0}\right)$ is a bit too large for a O7V star and would better fit to a slightly earlier main sequence O-type star, but it is compatible with an O7III type. However, the magnitudes given in Stasińska et al. (1986) and Oey \& Massey (1995) seem to favor a main sequence classification.

When trying to estimate the He II flux, we discovered an apparent continuum emission to the north of Star \#2 in our spectra. The He II image thus actually contains He II+[Ar IV]+cont., and the pollution due to these additional components is larger where the He II nebula is fainter. That is the reason why we choose to measure the He II flux only in the brightest parts of

\footnotetext{
${ }^{3}$ Most of the differences in $L(4686) / L(\beta)$ between Garnett et al. (1991b) and Garnett et al. (2000) - which were discussed in length in the latter - can be explained by this error. Further discrepancies can be explained by the fact that neither the He II nor the $\mathrm{H} \alpha$ nebula presents a spherical shape; that Stasińska et al. (1986) measured only the northern edge of the He II nebula and that there may be an error in the Stasińska et al. (1986) paper (Pakull \& Motch 1989a). We also note that our extinction $A_{\mathrm{V}}=0.55 \mathrm{mag}$, corresponding to $c_{\beta}=0.26$ and a color excess $E(B-V)=0.18 \mathrm{mag}$, is close to the average value found by Oey \& Massey (1995). It is thus possible that the extinction $A_{\mathrm{V}}=0.25 \mathrm{mag}$ of Garnett et al. (2000) actually refers to $c_{\beta}$. However, that fact alone can not explain the large discrepancy observed between the He II fluxes.
}

the nebula, i.e. a region of radius $7 "$ comprising the bright arc. Therefore we may miss part of the He II flux, but part of this error is compensated by the contamination of [Ar IV] + cont. We find $L($ He II 14686$)=4 \times 10^{35} \mathrm{erg} \mathrm{s}^{-1}$ and $Q\left(\mathrm{He}^{+}\right)=$ $4 \times 10^{47}$ photons $\mathrm{s}^{-1}$, after the reddening correction and for a distance of $50 \mathrm{kpc}$. In the past, a few estimates of the He II flux have been made: using the results of Stasińska et al. (1986), Garnett et al. (1991b) found $L($ He II $\lambda 4686)=6.3 \times$ $10^{35} \mathrm{erg} \mathrm{s}^{-1}$ for a distance of $57 \mathrm{kpc}$ (or $4.7 \times 10^{35} \mathrm{erg} \mathrm{s}^{-1}$ at a distance of $50 \mathrm{kpc}$ ) but their estimate was made considering a spherical geometry, which is obviously not the case. This approximation could lead to a large overestimate of the flux, but since Stasińska et al. (1986) did not measure the $I(\mathrm{He}$ II $\lambda 4686) / I(\mathrm{H} \beta)$ in the brightest part of the He II nebula, their result is probably not too far off. Using again Stasińska et al. (1986) measurements, Pakull \& Motch (1989a) quote a lower value $L$ (He II 14686 ) of $3 \times 10^{35} \mathrm{erg} \mathrm{s}^{-1}$, after "taking into account an apparent error in Stasińska et al. (1986) paper". Garnett et al. (2000) found $L(\mathrm{He}$ II 24686$)=2.2 \times 10^{35} \mathrm{erg} \mathrm{s}^{-1}$, which is lower than our value, but the region considered by these authors is not specified clearly, and their He II images, taken with the CTIO $0.9 \mathrm{~m}$ telescope, probably did not reach a high signal/noise (the authors actually did not show the images). However, since we took our spectra with the same slit orientation as in Garnett et al. (2000), we can compare rather the $I(\mathrm{He}$ II $\lambda 4686) / I(\beta)$ ratio. Garnett et al. (2000) quoted a value of $0.064 \pm 0.003$, and we find $0.061 \pm 0.004$ when considering like them the whole nebula, including Stars \# 1 \& 2. Furthermore, in a region similar and as close as possible to the position observed by Stasińska et al. (1986), we get similar results as these authors as well. We thus notice hardly any longterm change in the He II emission. 
Apparently, the He II emission of N44C is still a puzzle. The ionizing star(s) in the vicinity cannot provide enough hard photons and there is no high-velocity gas that could trace a shock capable of ionizing $\mathrm{He}^{+}$. The last hypothesis $-\mathrm{a}$ fossil X-ray nebula (Pakull \& Motch 1989a) - implies a recombination of He II, while we see no clear evidence for a change in the line intensity over the last 15 years. Recombination of [Ne IV] and $[\mathrm{Ne} \mathrm{V}]$ is also expected, but unfortunately, there are no lines of these ions in the wavelength ranges covered by our spectra. Pakull \& Motch (1989a) estimated recombination timescales of 20 and $100 \mathrm{yr}$ for [Ne V] and He II, respectively. Garnett et al. (2000) favored smaller values, of 5 and $20 \mathrm{yr}^{4}$. Since the proposed associated X-ray source, LMC X-5, was observed in 1974-76 by OSO7 and Ariel V, we should detect a significant reduction of the He II lines, even with a timescale as long as $100 \mathrm{yr}$. Apart from a fourth and still unknown ionizing mechanism, one possibility to explain the observations could be that the recombination timescale is much longer, thus that the density in N44C is very low. Another could be that the mysterious $\mathrm{X}$-ray source responsible of the ionization had recently turned on again, reionizing the nebula, but we note that Einstein data, a ROSAT pointing, and a recent Chandra observation of N44 do not reveal any source in the vicinity of N44C (Y.-H. Chu, private communication). It would be worth to follow the evolution of the He II, [Ne IV] and [Ne V] and X-ray emissions in the future, to determine the temporal behaviour of the nebula. The shape and the location of the He II emission strongly suggest that the phenomenon is associated in some way with Star \#2. Monitoring of this object should allow to detect signs of orbital motion, and thus the presence of the hypothetical compact companion needed for the fossil X-ray nebula scenario. However, we note that the $90 \%$ confidence error on the position of LMC X-5 is $\sim 0.3^{\circ}$ while $\mathrm{N} 44 \mathrm{C}$ lies at $0.5^{\circ}$ from the $\mathrm{X}$-ray source. We further note the absence of any evidence (in the X-ray or radio domains, by means of high velocity motions or enhanced $[\mathrm{S} \mathrm{II}] / \mathrm{H} \alpha$ ratio) of a supernova explosion in the vicinity of Star \#2 that could have given birth to a compact object.

\section{WR survey}

We have undertaken a small survey to search for He II emission around a few WR stars of the LMC. Similar surveys had previously been undertaken by Niemela et al. (1991) and Pakull (1991), mainly concentrating on WN stars. We chose seven other WR stars: BAT99-8, 9, 11, 52, 63, 84 and 134. None of them shows any sign of nebular He II emission, despite the fact that Melnick \& Heydari-Malayeri (1991) claimed to have found possible extended He II emission around BAT99-9. However, BAT99-8, 11, 63 and 134 are known to present ring nebulae around them, and we study here their physical properties, which can help to determine the evolutionary status of the WR bubbles. Chu et al. (1999) have suggested that the large size and surface brightness variations of the nebulae around

\footnotetext{
4 However, we note that Garnett et al. (2000) were puzzled by the strength of the observed [Ne IV] lines, incompatible with their short timescales.
}

BAT99-11, 63 and 134 support the existence of some interactions with the ISM but also that their large expansion velocities and the rather regular expansion pattern show that they are not yet dominated by the ISM.

\subsection{BAT99-8}

BAT99-8 (Brey 8, Sk-6942, HD 32257), a WC4 star (Bartzakos et al. 2001), is surrounded by a ring nebula of size $150^{\prime \prime} \times 230^{\prime \prime}$ (Dopita et al. 1994). Two faint, arcuate filaments are visible to the north and southeast of the star. They are more easily distinguished from the background nebula in the [O III] lines. Unfortunately, no kinematic study of this candidate windblown bubble is available, and its exact nature and expansion status are still unknown. We have analysed the spectra of each arc (15" long for the northern one and $19^{\prime \prime}$ for the southern one), and we present in Table 5 their dereddened line ratios.

No nebular He II emission was detected in this nebula, but the spectra still allow us to derive an upper limit on the strength of the He II emission by estimating the $3 \sigma$ level at the position of He II $\lambda 4686$. This leads to $F(\mathrm{He}$ II $\lambda 4686) / F(\mathrm{H} \beta)<0.007$ in the most restrictive case, i.e. the one that gives the lowest upper limit. Assuming a geometry composed of two partial rings of uniform brightness (a quarter of a ring of radius $65^{\prime \prime}$ and width $7 "$, plus one eighth of a ring of radius $111^{\prime \prime}$ and width 7 ", see the morphology of the $\mathrm{H} \alpha$ nebula in Fig. 1c of Dopita et al. 1994), a distance of $50 \mathrm{kpc}$ and a reddening $A_{\mathrm{V}}$ of $0.4 \mathrm{mag}$, we then find an upper limit on the ionization power of $\log \left[Q\left(\mathrm{He}^{+}\right)\right]<45.3$.

The faintness of the [O III] lines renders the determination of the temperature of the nebula rather difficult: in our sample, such weakness of the [O III] $\lambda \lambda$ 4959, 5007 lines compared to $\mathrm{H} \beta$ is only seen for the N44 superbubble. That may suggest a temperature of $10 \mathrm{kK}$ for this nebula. Such a low temperature is further supported by the rather strong [O II], [S II] and [N II] lines. For this temperature, we found only an oxygen abundance slightly higher than the LMC average. Higher temperatures of $12.5 \mathrm{kK}$ or $15 \mathrm{kK}$ could lead to significant departures from average, with a low oxygen abundance and a quite high N/O ratio, but such a temperature appears less probable.

\subsection{BAT99-11}

The nebula surrounding BAT99-11 (Brey 10, Sk-6815, HD 32402), a WC4 star (Bartzakos et al. 2001), was discovered quite a long time ago by Chu (1981). It has an elliptical shape of size $180^{\prime \prime} \times 112^{\prime \prime}$ and its surface brightness is far from uniform, with the southwestern region being the brightest (Dopita et al. 1994). A kinematic study reveals that this nebula corresponds to a shell expanding into a quiescent H II region with a velocity of $\sim 42 \mathrm{~km} \mathrm{~s}^{-1}$ (Chu et al. 1999). We have examined the spectra of both sides of the nebula ( $29^{\prime \prime}$ for the SW arc and $48^{\prime \prime}$ for the NE one), and present their line ratios in Table 5. As above, we estimated upper limits on the strength of the He II emission of this nebula and find $F(\mathrm{He}$ II $\lambda 4686) / F(\mathrm{H} \beta)<0.002$ in the most restrictive case. Assuming an elliptical ring geometry $\left(111^{\prime \prime} \times 183^{\prime \prime}\right.$ for the size 
Table 5. Same as Table 2 for LMC BAT99-8, 11, 63 and 134.

\begin{tabular}{|c|c|c|c|c|c|c|c|c|c|}
\hline & \multicolumn{2}{|c|}{ BAT99-8 ${ }^{a}$} & \multicolumn{2}{|c|}{ BAT99-11 } & \multirow{2}{*}{$\begin{array}{c}\text { BAT99-63 } \\
\text { NE }\end{array}$} & \multicolumn{4}{|c|}{ BAT99-134 } \\
\hline & $\mathrm{N}$ & $\mathrm{S}$ & $\mathrm{NE}^{b}$ & SW & & $\mathrm{NE}$ & near the star & SW & beyond $\mathrm{SW}^{c}$ \\
\hline [O II] 3727 & $752(78)$ & $602(63)$ & $449(47)$ & $367(38)$ & $220(23)$ & $61(6)$ & $238(25)$ & $197(21)$ & $463(48)$ \\
\hline $\mathrm{H} 10$ & & & & $5.9(0.6)$ & & & & & \\
\hline H9 & & & & $10(1)$ & & & & & \\
\hline [Ne III] 3868 & 12: & & & $4.2(0.4)$ & $61(6)$ & $106(10)$ & $84(8)$ & $78(8)$ & $104(10)$ \\
\hline $\mathrm{H} 8+\mathrm{He} \mathrm{I}$ & 15: & & $17(2)$ & $19(2)$ & $19(2)$ & $26(3)$ & $23(2)$ & $19(2)$ & 27: \\
\hline$[\mathrm{Ne} \mathrm{III}]+\mathrm{H} \epsilon$ & $24(2)$ & & $13(1)$ & $16(2)$ & $27(3)$ & $41(4)$ & $37(4)$ & $42(4)$ & $40(4)$ \\
\hline $\mathrm{H} \delta$ & $29(3)$ & 24: & $27(2)$ & $28(3)$ & $33(3)$ & $36(3)$ & $32(3)$ & $28(3)$ & $30(3)$ \\
\hline $\mathrm{H} \gamma$ & $46(4)$ & $41(3)$ & $50(4)$ & $51(4)$ & $49(4)$ & $51(4)$ & $48(4)$ & $51(4)$ & $50(4)$ \\
\hline [O III $] 4363$ & & & $4.9(0.4)$ & $3.7(0.3)$ & $5.1(0.4)$ & $19(2)$ & $12(1)$ & $10(1)$ & \\
\hline He I 4471 & & & $4.1(0.3)$ & $4.1(0.3)$ & $5.4(0.4)$ & & & $3.9:$ & \\
\hline $\mathrm{H} \beta$ & 100 & 100 & 100. & 100. & 100. & 100. & 100 & 100. & 100. \\
\hline [O III $] 4959$ & $61(4)$ & $60(4)$ & $78(6)$ & $132(9)$ & 237 (17) & $415(29)$ & $284(20)$ & $296(21)$ & $256(18)$ \\
\hline [O III $] 5007$ & $177(13)$ & $184(13)$ & $233(17)$ & $393(28)$ & $710(51)$ & $1246(89)$ & $846(60)$ & $890(63)$ & $770(55)$ \\
\hline He I 5876 & $9 .:$ & 12: & $12(1)$ & $13(1)$ & $13(1)$ & $13(1)$ & $15(1)$ & $11(1)$ & $12(1)$ \\
\hline [S III] 6312 & & & & $1.9(0.2)$ & $2.3(0.2)$ & & & & \\
\hline [N II] 6548 & $21(2)$ & $20(2)$ & $7.8(0.8)$ & $5.7(0.6)$ & 6. (0.6) & & $9.1(0.9)$ & $5.1(0.5)$ & $12(1)$ \\
\hline $\mathrm{H} \alpha$ & $286(29)$ & $286(29)$ & $279(28)$ & $282(28)$ & $286(29)$ & $282(28)$ & $282(28)$ & $282(28)$ & $282(28)$ \\
\hline [N II] 6583 & $52(5)$ & $45(5)$ & $26(3)$ & $19(2)$ & $19(2)$ & 4.9: & $23(2)$ & $17(2)$ & $40(4)$ \\
\hline He I 6678 & & & $2.7(0.3)$ & $3.1(0.3)$ & $2.7(0.3)$ & & 4.: & 3.6: & \\
\hline [S II] 6716 & $87(9)$ & $72(7)$ & $20(2)$ & $13(1)$ & $24(2)$ & 6.5: & $34(4)$ & $25(3)$ & $61(6)$ \\
\hline [S II] 6731 & $65(7)$ & $56(6)$ & $15(2)$ & $9.4(1)$. & $17(2)$ & 8.0: & $25(3)$ & $18(2)$ & $43(4)$ \\
\hline$F(\mathrm{H} \beta)\left(10^{-14} \mathrm{erg} \mathrm{cm}^{-2} \mathrm{~s}^{-1}\right)$ & 0.85 & 0.44 & 4. & 8.7 & 4.1 & 0.64 & 1.2 & 0.86 & 1. \\
\hline$A_{\mathrm{V}}(\mathrm{mag})$ & 0.48 & 0.28 & 0.59 & 0.58 & 0.35 & 0.28 & 0.22 & 0.34 & 0.34 \\
\hline$T_{\mathrm{e}}[\mathrm{O} \mathrm{III}](\mathrm{kK})$ & & & $15.6 \pm 0.8$ & $11.4 \pm 0.4$ & $10.4 \pm 0.3$ & $13.6 \pm 0.6$ & $13.1 \pm 0.6$ & $12.1 \pm 0.5$ & \\
\hline$n_{\mathrm{e}}[\mathrm{S} \mathrm{II}]\left(\mathrm{cm}^{-3}\right)$ & $<320$ & $<410$ & $<380$ & $<300$ & $<280$ & & $<290$ & $<270$ & $<260$ \\
\hline $\mathrm{He}^{+} / \mathrm{H}^{+} \times 10^{2}$ & $6.5:$ & $8.6:$ & $8.3 \pm 0.4$ & $8.9 \pm 0.5$ & $8.9 \pm 0.4$ & $10 . \pm 0.9$ & $11 \pm 0.5$ & $8.5 \pm 0.7$ & $8.8 \pm 0.8$ \\
\hline $\mathrm{O}^{+} / \mathrm{H}^{+} \times 10^{5}$ & $28 \pm 3$ & $23 \pm 2$ & $3.4 \pm 0.4$ & $8 . \pm 0.8$ & $7 . \pm 0.7$ & $7.1 \pm 0.7$ & $3.1 \pm 0.3$ & $3.5 \pm 0.4$ & $7.2 \pm 0.8$ \\
\hline $\mathrm{O}^{2+} / \mathrm{H}^{+} \times 10^{5}$ & $6.3 \pm 0.4$ & $6.3 \pm 0.3$ & $2.2 \pm 0.1$ & $9 . \pm 0.4$ & $22 \pm 1$ & $17 \pm 1$ & $13 \pm 1$ & $17 \pm 1$ & $13 \pm 1$ \\
\hline$\rightarrow \mathrm{O} / \mathrm{H} \times 10^{4}$ & $3.5 \pm 0.3$ & $2.9 \pm 0.2$ & $0.56 \pm 0.04$ & $1.7 \pm 0.1$ & $2.9 \pm 0.1$ & $1.8 \pm 0.1$ & $1.6 \pm 0.1$ & $2 . \pm 0.1$ & $2 . \pm 0.1$ \\
\hline $\mathrm{N}^{+} / \mathrm{H}^{+} \times 10^{6}$ & $11 \pm 1$ & $10 \pm 1$ & $1.8 \pm 0.1$ & $2.5 \pm 0.2$ & $3.2 \pm 0.2$ & $0.5:$ & $2.6 \pm 0.2$ & $1.9 \pm 0.1$ & $4.4 \pm 0.3$ \\
\hline$\left(\rightarrow \mathrm{N} / \mathrm{O} \times 10^{2}\right)$ & $3.9 \pm 0.5$ & $4.6 \pm 0.6$ & $5.3 \pm 0.7$ & $3.2 \pm 0.4$ & $4.6 \pm 0.6$ & 6.6: & $8.2 \pm 1$ & $5.6 \pm 0.7$ & $6.1 \pm 0.8$ \\
\hline $\mathrm{S}^{+} / \mathrm{H}^{+} \times 10^{7}$ & $35 \pm 3$ & $30 \pm 2$ & $3.2 \pm 0.2$ & $3.8 \pm 0.3$ & $8.7 \pm 0.6$ & 1.8: & $7.4 \pm 0.5$ & $6.4 \pm 0.5$ & $15 \pm 1$ \\
\hline $\mathrm{S}^{2+} / \mathrm{H}^{+} \times 10^{6}$ & & & & $2.8 \pm 0.3$ & $4.8 \pm 0.5$ & & & & \\
\hline $\mathrm{Ne}^{2+} / \mathrm{H}^{+} \times 10^{5}$ & 1.3: & & & $0.28 \pm 0.03$ & $5.7 \pm 0.6$ & $3.9 \pm 0.4$ & $3.5 \pm 0.3$ & $4.2 \pm 0.4$ & $5 . \pm 0.5$ \\
\hline
\end{tabular}

${ }^{a}$ Assuming $T_{\mathrm{e}}=10 \mathrm{kK}$

${ }^{b}$ Abundances shown below were derived for $T_{\mathrm{e}}=T([\mathrm{O} \mathrm{III}])=15.6 \mathrm{kK}$.

${ }^{c}$ Assuming $T_{\mathrm{e}}=12.5 \mathrm{kK}$ (close to the temperatures of the neighbouring regions). 
of the external ellipse, and $85^{\prime \prime} \times 144^{\prime \prime}$ for the internal one) and a reddening $A_{\mathrm{V}}$ of $0.58 \mathrm{mag}$, we then find $\log \left[Q\left(\mathrm{He}^{+}\right)\right]<46.2$.

The large temperature derived for the fainter northeastern region is probably due to the rather low $\mathrm{S} / \mathrm{N}$ in the [O III] $\lambda 4363$ line, and is thus to consider with caution. The most reliable abundances, derived for the brightest side of the nebula, only show $\mathrm{O} / \mathrm{H}$ and $\mathrm{N} / \mathrm{O}$ values slightly lower than the LMC values. Similar results are found for the NE region when using the temperature of the SW one, whilst a normal helium abundance and N/O ratio, but a very low oxygen abundance are found for the higher temperature derived from the [O III] lines. Like for BAT99-2, we may be observing a circumstellar bubble merging with the interstellar one.

\subsection{BAT99-63}

A fine filamentary ring nebula, some $65^{\prime \prime} \times 88^{\prime \prime}$ in size, surrounds the star BAT99-63 (Brey 52, classified WN4ha: in Foellmi et al. 2003b). The southwestern part of the ring is the faintest, while the northeastern region is much brighter. The analysis of the kinematics of this nebula suggests a blister structure, with an expansion velocity of $\sim 50 \mathrm{~km} \mathrm{~s}^{-1}$ in the low density ISM (Chu et al. 1999). We studied the spectrum of the brightest region, situated to the NE of the star (62" long, see Table 5). Using the same method as for BAT99-8 and 11, we find an upper limit on the He II emission of $F(\mathrm{He}$ II $\lambda 4686) / F(\mathrm{H} \beta)<0.006$ in the most restrictive case. Assuming an elliptical ring geometry $\left(80^{\prime \prime} \times 70^{\prime \prime}\right.$ and $58^{\prime \prime} \times 45^{\prime \prime}$ for the sizes of the external and internal ellipses, respectively) and a reddening $A_{\mathrm{V}}$ of $0.4 \mathrm{mag}$, we then find $\log \left[Q\left(\mathrm{He}^{+}\right)\right]<45.4$. Even if the oxygen abundance might be slightly larger than the LMC average, no significant chemical enrichment can be detected in this nebula, favoring the hypothesis of an interstellar bubble already dominated by the ISM.

\subsection{BAT99-134}

A beautiful ring nebula of size $87^{\prime \prime} \times 107^{\prime \prime}$ (Dopita et al. 1994) completely surrounds BAT99-134 (Brey 100, Sk-67268, HD 270149), a WN4b star (Foellmi et al. 2003b). Its southeastern side is brighter, and the WR star appears largely decentred. Using high dispersion spectra, Chu (1983) detected an expansion velocity of $45-50 \mathrm{~km} \mathrm{~s}^{-1}$ in this nebula. The same author proposed that it is actually a small shell expanding on the surface of a molecular cloud. She further suggested that the motion of the WR star, probably originally formed on the cloud's surface, had caused the offset. We decided to analyse four regions in this nebula: the first and second sample the NE and SW part of the ring (18" and $15^{\prime \prime}$ long, respectively), the third covers the region situated between the star and the SW arc (47" long), and the last one studies the H II region beyond the SW arc (46" long), that belongs to a larger shell unrelated to the WR wind-blown bubble. Compared to the LMC average values, the regions of the nebula closer to the star and the NE arc appear slightly enriched in helium and depleted in oxygen. The $\mathrm{N} / \mathrm{O}$ ratio is also generally larger. These abundance differences fit well the Chu (1983) model: the southwesternmost part of the wind-blown bubble and the outer H II region actually belong to the molecular cloud's edge, and should thus present normal abundances.

Using the same method as above, we find an upper limit on the He II emission of $F(\mathrm{He}$ II $\lambda 4686) / F(\mathrm{H} \beta)<0.007$ in the most restrictive case. Assuming an elliptical ring geometry $\left(80^{\prime \prime} \times 100^{\prime \prime}\right.$ and $66^{\prime \prime} \times 79^{\prime \prime}$ for the sizes of the external and internal ellipses, respectively) and a reddening $A_{\mathrm{V}}$ of $0.3 \mathrm{mag}$, we then find $\log \left[Q\left(\mathrm{He}^{+}\right)\right]<45.5$.

\subsection{WR stars and nebular He II emission: Discussion}

Up to now, only 3 WR stars of the MCs are known to display nebular He II emission: BAT99-2, 49 and AB7, while 32 others (BAT99-5, 6, 8, 9, 11, 12, 15, 19, 29, 31, 32, 36, 38, 43, 52, 53, $56,59,60,62,63,64,81,82,84,92,94,123,124,126,132$ and 134, Pakull 1991, Niemela 1991, and this paper) have none. For LMC WC4 stars, including our studied cases of BAT99-8, 9, 11 and 52, Gräfener et al. (1998) and Crowther et al. (2002) estimated temperatures $T_{\star}$ of $\sim 85-100 \mathrm{kK}$. Following the models of Smith et al. (2002), this would not produce any detectable He II nebula since $Q\left(\mathrm{He}^{+}\right)<2-5 \times 10^{40}$ photons s$^{-1}$ for $T_{\star} \leq 100 \mathrm{kK}$ WC stars at $Z=0.2-0.4 Z_{\odot}$. The non dectection and/or upper limits of the He II $\lambda 4686$ line around the aforementioned WC4 stars agree thus completely with the latest models.

However the situation appears more complex for WN stars. The WN2 star BAT99-5 has a spectrum almost identical to BAT99-2 (Foellmi et al. 2003b), but no He II emission was detected in its surroundings $(F(\mathrm{He}$ II $\lambda 4686) / F(\mathrm{H} \beta)<0.04$ in Pakull 1991). Hamann \& Koesterke (2000) fitted the spectrum of BAT99-5 with a temperature of $71 \mathrm{kK}$, a value too low to produce enough $\mathrm{He}^{+}$ionizing photons. We note however that in the case of the Galactic WN2 star, WR2, Hamann \& Koesterke (1998) found $T_{\star}=141 \mathrm{kK}$, a temperature much larger than for BAT99-5. This is rather remarkable since the average temperature of all other WN subtypes is rather similar in the Galaxy and the LMC (Hamann \& Koesterke 1998; Hamann \& Koesterke 2000; Crowther et al. 1995a; Crowther et al. 1995b; Crowther \& Smith 1997), though with a large scatter in both cases. Logically, using all information available, both stars, BAT99-2 and 5, or none of them should exhibit nebular He II emission.

Large discrepancies are also seen amongst WN4 stars. Hamann \& Koesterke (2000) found temperatures of 71 to $100 \mathrm{kK}$ for the LMC WN4 stars: the hottest of these stars should thus be capable of ionizing $\mathrm{He}^{+}$. For example, BAT99-94 (Brey 85) has a temperature of $100 \mathrm{kK}$, but yet no nebular He II was ever detected around this star (Niemela et al. 1991), contrary to BAT99-49. Moreover, the WN3 stars should in principle be hotter than the WN4 stars, but no nebular He II emission has been detected around BAT99-62 and $82(F(\mathrm{He}$ II $\lambda 4686) / F(\mathrm{H} \beta)<0.03$ and 0.007 , respectively, in Pakull 1991; Niemela et al. 1991).

Finally, we note that in the remaining cases of He II nebulae excited by WR stars (WR 102 in the Galaxy, IC 1613\#3 in IC 1613), these stars present a WO 
spectral type. However, near the only WO star of the LMC, BAT99-123 (Brey 93), no nebular He II emission was detected $\left(F\left(\mathrm{He}_{\mathrm{II}} \lambda 4686\right) / F(\mathrm{H} \beta)<0.07\right.$ in Pakull 1991). Even in the case of the largest concentration of hot stars in the LMC, i.e. in the 30 Doradus complex, no nebular He II emission was ever reported.

BAT99-2, 49 and AB7 seem thus different from all other WR stars of the MCs, but we find no particular reason for this uniqueness in the available data. Up to now, only $3 \mathrm{He}^{+}$excitation mechanisms have been proposed: high velocity shocks - but we are not aware of any peculiar motions near these objects; X-ray ionization - but no bright X-ray source was ever recorded in the close neighbourhood of any of these stars; photoionization - but then why would these stars differ from (very) similar WN stars of the same Galaxy? Could binarity play a role? AB7 and BAT99-49 indeed have massive companions, but BAT99-2 appears single (Foellmi et al. 2003b). Could they be mainly fossil He II nebulae? Long-term monitoring of both the nebulae and the stars would be required to unveil the signs of recombination as well as the orbital motion due to the presence of a putative compact companion in a wide eccentric orbit. However, up to now, only the visible spectrum of these peculiar stars is well studied, but the latter constitutes only a small part of the hot stars' radiation. The UV and X-ray fluxes of the WR stars of the MCs should be investigated deeply, since these peculiar stars may present particular features in these energy ranges.

\section{Conclusion}

In this paper and a previous letter (Paper I), we investigate the relations between hot stars of the MCs and their environment. We first focus on the peculiar He II nebulae ionized by WR stars. We provide here the first high quality He II $\lambda 4686$ images of these high excitation nebulae. The He II nebula around BAT99-49 appears more extended but also fainter than the one associated with BAT99-2. The high excitation nebula inside N76 occupies a size of roughly half the one of the $\mathrm{H} \alpha$ nebula. It is centered on the WR star AB7. Once calibrated, the images enable us to estimate the $\mathrm{He}^{+}$ionizing fluxes. For BAT99-2 and 49, we found $Q\left(\mathrm{He}^{+}\right)$of 4 and $1 \times 10^{47}$ photons s $^{-1}$, respectively. In the latest theoretical models, this corresponds to $90-100 \mathrm{kK}$ WN stars. AB7 apparently emits $Q\left(\mathrm{He}^{+}\right)=5 \times 10^{48}$ photons s ${ }^{-1}$, and no recent model could explain such a high value. In addition, after comparing these objects to other WR stars of the MCs, we find no particular reason for their uniqueness: other very similar stars do not present such high excitation features.

In this study, we also used FORS spectroscopy to search for He II emission around seven other WR stars: BAT99-8, 9, $11,52,63,84$ and 134. We do not find any new He II nebula, even near BAT99-9 where hints of nebular He II emission had been found in a previous study. These data enable us to analyse the physical properties of the above nebulae and also of the ring nebulae associated with BAT99-8, 11, 63 and 134. The abundance determinations show only small chemical enrichment for the nebulae around BAT99-2, 49, 100 and AB7. The slightly larger N/O and the slightly lower oxygen abundance are incompatible with a single circumstellar bubble, but could be explained in a scenario where the circumstellar bubble blown by the WR star is currently merging with the interstellar bubble blown by its progenitor. In contrast, N44C and the nebulae associated with BAT99-8 and 63 present normal abundances, suggesting ISM dominated bubbles.

We also examine a fourth He II nebula of the MCs, N44C. It is thought to be a fossil X-ray nebula in which He II should recombine with timescales of 20-100 yr. Using our VLT data, we estimate $L(\mathrm{H} \beta)=9 \times 10^{36} \mathrm{erg} \mathrm{s}^{-1}$ and $L(\mathrm{He}$ II $\lambda 4686)=4 \times 10^{35} \mathrm{erg} \mathrm{s}^{-1}$. Comparing our data with older values, we find no sign of variation in the He II $\lambda 4686$ line strength. This puzzling result indicates a much longer recombination timescale, and thus a very low density. Alternatively, another, unknown, ionizing mechanism could be at work. The X-ray source may also have turned on again some time in between the previous observations and ours. Further monitoring is recommended to follow the possible evolution of the He II and X-ray emissions, and also to search for traces of orbital motion in Star\#2, and thus of a companion that would be responsible for the episodic X-ray emission as required by the fossil X-ray nebula scenario.

Note added in proof: Pakull (1989) reported radial velocity variations of Star\#2 in N44C that may indicate the presence of a massive companion. However, new observations are needed in order to confirm this result and to determine the exact nature of the companion.

Acknowledgements. We acknowledge support from the PRODEX $\mathrm{XMM}-\mathrm{OM}$ and Integral Projects and through contracts $\mathrm{P} 4 / 05$ and P5/36 "Pôle d'attraction Interuniversitaire" (Belgium). We thank the referee, Dr. M. Heydari-Malayeri, for his useful suggestions.

\section{References}

Bartzakos, P., Moffat, A. F. J., \& Niemela, V. S. 2001, MNRAS, 324, 18

Benjamin, R. A., Skillman, E. D., \& Smits, D. P. 1999, ApJ, 514, 307

Bica, E. L. D., Schmitt, H. R., Dutra, C. M., \& Oliveira, H. L. 1999, AJ, 117, 238

Bouchet, P., Lequeux, J., Maurice, E., Prévot, L., \& Prévot-Burnichon, M. L. 1985, A\&A, 149, 330

Caplan, J., \& Deharveng, L. 1985, A\&AS, 62, 63

Cardelli, J. A., Clayton, G. C., \& Mathis, J. S. 1989, ApJ, 345, 245

Chu, Y.-H. 1981, Ph.D. Thesis, University of California, Berkeley

Chu, Y.-H. 1983, ApJ, 269, 202

Chu, Y.-H., Weis, K., \& Garnett, D. R. 1999, AJ, 117, 1433

Crowther, P. A., Hillier, D. J., \& Smith, L. J. 1995a, A\&A, 293, 403

Crowther, P. A., Hillier, D. J., \& Smith, L. J. 1995b, A\&A, 302, 457

Crowther, P. A., \& Smith, L. J. 1997, A\&A, 320, 500

Crowther, P. A., Dessart, L., Hillier, D. J., Abbott, J. B., \& Fullerton, A. W. 2002, A\&A, 392, 653

Dopita, M. A., Bell, J. F., Chu, Y.-H., \& Lozinskaya, T. A. 1994, ApJS, 93, 455

Dopita, M. A., \& Hua, C. T. 1997, ApJS, 108, 515

Dufour, R. J. 1975, ApJ, 195, 315

Fitzpatrick, E. L. 1989, IAUS, 135, 37

Foellmi, C., Moffat, A. F. J., \& Guerrero, M. A. 2003a, MNRAS, 338, 360 
Foellmi, C., Moffat, A. F. J., \& Guerrero, M. A. 2003b, MNRAS, 338, Niemela, V. S., Massey, P., Testor, G., \& Giménez-Benítez, S. 2002, 1025 MNRAS, 333, 347

García-Segura, G., Langer, N., \& MacLow, M.-M. 1996, A\&A, 316, 133

Oey, M. S., \& Massey, P. 1995, ApJ, 452, 210

Oke, J. B. 1990, AJ, 99, 1621

Garnett, D. R., Kennicutt, R. C. Jr., Chu, Y.-H., \& Skillman, E. D. 1991a, PASP, 103, 850

Garnett, D. R., Kennicutt, R. C. Jr., Chu, Y.-H., \& Skillman, E. D. 1991b, ApJ, 373, 458

Garnett, D. R., Galarza, V. C., \& Chu, Y.-H. 2000, ApJ, 545, 251

Goudis, C., \& Meaburn, J. 1984, A\&A, 137, 152

Gräfener, G., Hamann, W.-R., Hillier, D. J., \& Koesterke, L. 1998, A\&A, 329, 190

Hamann, W.-R., \& Koesterke, L. 1998, A\&A, 333, 251

Hamann, W.-R., \& Koesterke, L. 2000, A\&A, 360, 647

Pakull, M. W. 1989, Recent Developments of Magellanic Cloud Research, ed. K. S. de Boer, F. Spite, \& G. Stasinska (ParisMeudon), 183

Pakull, M. W., \& Motch, C. 1989a, Nature, 337, 337

Pakull, M. W., \& Motch, C. 1989b, Extranuclear Activity in Galaxies, ed. E. J. A. Meurs, \& R. A. E. Fosbury (Garching bei Munchen), 285

Pakull, M. W., \& Bianchi, L. 1991, IAUS, 143, 260

Pakull, M. W. 1991, IAUS, 143, 391

Russell, S. C., \& Dopita, M. A. 1990, ApJS, 74, 93

Heydari-Malayeri, M., Charmandaris, V., Deharveng, L., et al. 2001, A\&A, 372, 527

Schaerer, D. 1996, ApJ, 467, L17

Shaw, R. A., \& Dufour, R. J. 1995, PASP, 107, 896

Massey, P., Waterhouse, E., \& Degioia-Eastwood, K. 2000, AJ, 119, 2214

McCall, M. L., Rybski, P. M., \& Shields, G. A. 1985, ApJS, 57, 1

Melnick, J., \& Heydari-Malayeri, M. 1991, IAUS, 143, 409

Nazé, Y., Chu, Y.-H., Points, S. D., et al. 2001, AJ, 122, 921

Nazé, Y., Chu, Y.-H., Guerrero, M. A., et al. 2002, AJ, 124, 3325

Nazé, Y., Rauw, G., Manfroid, J., Chu, Y.-H., \& Vreux, J.-M. 2003, A\&A, 401, L13 (Paper I)

Niemela, V. S., Heathcote, S. R., \& Weller, W. G. 1991, IAUS, 143, 425

Smith, L. J., Norris, R. P. F., \& Crowther, P. A. 2002, MNRAS, 337, 1309

Stasińska, G., Testor, G., \& Heydari-Malayeri, M. 1986, A\&A, 170, L4

Storey, P. J., \& Hummer, D. G. 1995, MNRAS, 272, 41

Tuohy, I. R., \& Dopita, M. A. 1983, ApJ, 268, L11

Walborn, N. R. 2002, Hot Star Workshop III: The Earliest Stages of Massive Star Birth, ed. P. A. Crowther (San Francisco), ASP Conf. Proc., 267, 111 